\title{
Beam-domain hybrid time-switching and power-splitting SWIPT in full-duplex massive MIMO system
}

\author{
Kui Xu, Zhexian Shen*, Yurong Wang and Xiaochen Xia
}

\begin{abstract}
In this paper, we consider the beam-domain hybrid time-switching (TS) and power-splitting (PS) simultaneous wireless information and power transfer (SWIPT) protocol design in full-duplex (FD) massive multiple-input multiple-output (MIMO) system, where the FD base station (BS) simultaneously serves a set of downlink half-duplex (HD) users (cellular users) and a set of fixed uplink HD users (fixed sensor nodes) which are uniformly distributed in its coverage area. In order to reduce the computational complexity, we investigate the beam-domain representation of massive MIMO channels based on the basis expansion model, and then the beam-domain SWIPT protocol which lies in intelligently scheduling the users and sensors based on the beam-domain distributions of their associated channels to mitigate $\mathrm{SI}$ and enhance transmission efficiency is designed for full-duplex massive MIMO system. The whole beam-domain hybrid TS and PS SWIPT protocol is divided into two phases based on the ideal of TS. The first phase is used for cellular users uplink training and sensor nodes energy harvesting as well as downlink training, wherein the cellular users transmit uplink pilots for uplink channel estimation at the BS, while the BS simultaneously transmits energy signals to the sensor nodes. Based on the idea of PS, the sensor nodes utilize the received energy signals for energy harvesting and downlink channel estimation. In the second phase, the BS forms the transmit beamformers for information transmission to the users as well as the receive beamformers for the sensors transmit their data to the BS simultaneously. By optimizing the TS ratio and transmit powers at the BS in two phases, the system achievable sum rate performance is maximized. Simulation results show the superiority of the proposed protocol on spectral efficiency compared with the existing massive MIMO SWIPT protocol.
\end{abstract}

Keywords: Full-duplex massive MIMO, Beam-domain channel representation, Simultaneous wireless information and power transfer, Beamforming, Achievable sum rate

\section{Introduction}

In the existing fourth generation (4G) system, multipleinput multiple-output (MIMO) technology has been used to improve the spectral efficiency (SE) performance. By deploying $M$ antennas at transmitter, and $N$ antennas at receiver, MIMO takes advantage of the spatial multiplexing gain of $\min (M, N)$ to improve the date rate. With the exponential growth of mobile Internet, the data traffic will increase by 1000 times beyond 2020. The existing $4 \mathrm{G}$ system cannot meet the increasing traffic demand of the future mobile communication, which put forward

*Correspondence: 18805198368@139.com

Institute of Communication Engineering, Army Engineering University of PLA, No. 2, Biaoying Street, Nanjing, China a great challenge to the fifth generation (5G) wireless communication systems [1].

As one of the key technologies in 5G, massive MIMO $[1,2]$, which uses large-scale antenna arrays (with 100 or more antennas) to replace the currently adopted multiple antennas, has been proved to be able to greatly improve the SE of wireless communications system [3-5]. As the number of antennas in massive MIMO grows larger, the simple linear precoder and detector tend to optimize, and the noise as well as the uncorrelated interference can be effectively eliminated $[1,2]$. What is more, the spatial resolution of massive MIMO is remarkably improved when compared with the conventional MIMO, and the signal 
beam can be focused within a narrow range; hence, various kinds of interference can be substantially suppressed $[4,6-10]$.

In the conventional half-duplex (HD) systems, i.e., timedivision duplex (TDD) systems or frequency-division duplex (FDD) systems, the uplink and downlink are separated by orthogonal time or frequency resources, hence theoretically wastes half of the time-frequency resource [11]. As a potential SE enhancement technique in 5G systems, full-duplex (FD) wireless transmission scheme allows the transceiver to use the same frequency resource for simultaneous transmission and reception [12-16]. FD technology can potentially double the transmission capability of the wireless physical layer. In terms of practical FD implementation, the effect of self-interference (SI) due to the coupling of own high-powered transmit signals to the receiver must be reduced [11]. Thanks to the promising analog/digital and spatial domain SI cancelation techniques that can achieve high transmit-receive isolation which have been reported in the recent literatures $[11,17]$, now, FD is becoming feasible in the near future.

\subsection{Motivation and related work}

In addition to the SE, energy efficiency (EE) also has gained wide research attention for the design of wireless networks $[18,19]$. Energy constraints introduce an upper limit on the power of transmission and associated signal processing in wireless devices, especially for batterypowered equipments [20]. As a sustainable solution to maintain the lifetime of energy constrained wireless networks, energy harvesting $(\mathrm{EH})$ technique has recently received significant attention [21-23]. EH technology can effectively solve the charging problem in battery-powered wireless networks, wherein the wireless nodes are inaccessible or a large number of wireless nodes are widely distributed.

Various sources of renewable energy, such as ambient heat, wind, solar, and vibration, can be used to power wireless communication devices [24]. However, one common problem of collecting energy from such sources is its random nature. Energy availability variations with location, time, or environmental conditions make resource allocation in such systems very challenging. Radio frequency (RF) signals radiated by ambient transmitters can be identified as a viable new inspiration for $\mathrm{EH}$. Compared with conventional energy sources, RF signals can carry both information and energy simultaneously. Wireless devices collecting energy of RF signals to power the signal transmission are named as simultaneous wireless information and power transfer (SWIPT), which has recently attracted great attention for various wireless channels [25-28]. Owing to its features, SWIPT can be used to remotely power a variety of applications such as wireless sensor networks, body area networks, wireless charging facilities, and future cellular networks. Recently, several important advances of SWIPT in the antenna and RF EH circuit designs, have greatly increased the feasibility of $\mathrm{EH}$ in practical wireless applications [29-31].

The SWIPT receiver was originally designed for simultaneous information decoding (ID) and EH from the same received signal $[32,33]$, which is found to be unrealistic in the later research [34]. Due to the non-ideal EH circuit design, part of the RF signal energy will be lost during the ID process [35]. Hence, practical circuits used for EH are not able to decode the information carried by the signal directly. In the following SWIPT receiver designs, antennas with different functionalities are equipped for the information transceiver and the energy harvester, respectively. In [36], antennas with different power sensitivities are designed for receivers of different uses, i.e., -20 to $-10 \mathrm{dBm}$ for $\mathrm{EH}$ and $-60 \mathrm{dBm}$ for ID.

According to the signal partition method for EH and ID, there are mainly two types of practical SWIPT receiver designs, namely the time-switching (TS) and the powersplitting (PS) receiver architectures [18]. The TS receiver alternately switches between $\mathrm{EH}$ and ID according to the TS ratio [36]. The received RF signal is first sent to the $\mathrm{EH}$ receiver and then to the information receiver. In the PS receiver, the power of the incoming signal is split into two streams according to the PS ratio [26]. One portion of the received signal is sent to the $\mathrm{EH}$ receiver, and the remaining portion is sent to the information receiver.

The design of transceiver for MISO interference channel with EH is studied in [37], where the PS method is adopted and the transmit beamforming vectors as well as receive PS ratios are jointly optimized. The authors in $[38,39]$ consider a one-way single-antenna amplifyand-forward (AF) SWIPT relay networks, where both the TS and PS protocols are studied. The joint transceiver designs for multiuser MISO relay systems with EH is studied in [40], where the received signal is divided into two parts for ID and EH based on the PS principle. Since the two-way relaying (TWR) system can further improve the SE, many works also studied the SWIPT protocols in the TWR scenario. The authors in [41] considered a twoway AF relaying system with SWIPT, where an EH relay node is used to help two source nodes exchange information. In [42], the authors consider the sum-throughput maximization problem in TWR network, where all the nodes are wireless-powered. The authors in [43] consider a SWIPT AF TWR network, where two source nodes harvest energy from multiple relay nodes.

The above works on SWIPT relay network are studied in HD scenario, where devices can either transmit or receive on a single frequency band, but not simultaneously. Since FD can potentially double the wireless physical layer capacity, recently, the transceiver design problem in FD SWIPT systems has arised. In [44], the joint 
optimization problem of relay beamforming, the receiver PS ratio as well as the transmit power at the sources are investigated for SWIPT system with a FD MIMO AF relay to maximize the system achievable sum rate. The design of robust nonlinear transceivers for MIMO FD wireless-powered relay networks is studied in [45], where the effects of imperfect channel state information (CSI) is took into consideration. The joint transceiver design for a FD cloud radio access network with SWIPT is considered in [46]. The joint transceiver designs for FD K-Pair MIMO interference channel with SWIPT is considered in [47]. Robust secure beamforming scheme for wireless-powered FD MIMO systems with self-energy recycling is studied in [48].

Despite the previous works on EH in FD system or MIMO system, a few works have been done on the SWIPT protocol design in cellular system with massive MIMO base station (BS). In [21], the joint FD selfbackhaul and EH protocol for small cell networks with massive MIMO is investigated. In [49], SWIPT protocol for three-dimensional (3D) massive MIMO system is designed, where the matched filter (MF) precoder is adopted at the BS. In [50], the SWIPT protocol is designed for multipair TWR system with massive MIMO, where the linear precoders, i.e., zero-forcing (ZF) and maximal ratio combining (MRC), are adopted at the relay. A lowcomplexity SWIPT scheme with retrodirective maximum ratio transmission (MRT) beamforming is studied in [51], where all energy receivers (ERs) send a common beacon signal simultaneously to the energy transmitter (ET) in the uplink and the ET simply conjugates and amplifies its received sum-signal and transmits to all ERs in the downlink for SWIPT. For SWIPT system with massive MIMO, instantaneous full dimensional CSI is needed to perform linear precoding. To obtain full dimensional CSI, the uplink training overhead scales linearly with the number of user equipments (UE) in TDD system, the downlink training overhead scales linearly with the number of antennas in FDD system, and the corresponding CSI feedback yield an unacceptably high overhead, and therefore poses a significant bottleneck on the achievable SE [52].

\subsection{Contributions}

In this paper, we consider the beam-domain hybrid TS and PS SWIPT protocol design in FD massive MIMO system, where the FD BS simultaneously serves a set of downlink HD users (cellular users) and a set of fixed uplink HD users (fixed sensor nodes) which are uniformly distributed in its coverage area.

The contributions of this paper are summarized as follows:

- We investigate the beam-domain (BD) representation of massive MIMO channels based on the basis expansion model (BEM). In this way, the full dimensional channel can be compressed into BD effective channel (BDEC), as a result, the computational and implementation complexity can be greatly reduced and the SE performance can be improved.

- The BD hybrid TS and PS SWIPT protocol which lies in intelligently scheduling the users and sensors based on the BD distributions of their associated BDECs to mitigate SI and enhance SE performance is designed for FD massive MIMO system. Specifically, the whole BD hybrid TS and PS SWIPT protocol is divided into two phases based on the ideal of TS. Phase $I$ is used for cellular users uplink training and sensor nodes EH as well as downlink training. During this phase, the cellular users transmit pilots for uplink UE-BS channel estimation at the BS, while the BS simultaneously transmits energy signals to the sensor nodes. Based on the idea of PS, the sensor nodes utilize the received energy signals for $\mathrm{EH}$ and downlink Sensor-BS channel estimation. In phase II, the BS forms the transmit beams for information transmission to the users as well as the receive beams for the sensors transmit their data to the BS simultaneously.

- By optimizing the TS ratio and transmit powers at the BS in two phases, the system achievable sum rate performance is maximized. Simulation results shown the superiority of the proposed protocol on spectral efficiency compared with the conventional HD massive MIMO SWIPT protocol.

The rest of the paper is organized as follows. The system model is described in Section 2. Sections 3 and 4 consider the beam-domain channel representation and proposed BD massive MIMO FD SWIPT transmission scheme, respectively. Section 5 consider the practical beam-domain user and sensor grouping problem. Section 6 derives the system achievable sum rate and the optimum TS ratio as well as transmit powers at the BS in two phases. Section 7 presents the simulation results. Section 8 draws the conclusions.

Notations: In this paper, $\mathbb{E}(\cdot)$ denotes the expectation. $\mathbf{A}^{\left\{B_{1}, B_{2}\right\}}$ denotes the submatrix of $\mathbf{A}$ by keeping its rows indexed by set $B_{1}$ and columns indexed by set $B_{2}$. $\mathbf{A}^{\{B,:\}}$ and $\mathbf{A}^{\{: B\}}$ denote the submatrix of $\mathbf{A}$ by keeping its rows and columns indexed by set $B$, respectively. $\delta(\cdot)$ denotes the dirac delta function. $(\cdot)^{T},(\cdot)^{*},(\cdot)^{H},|\cdot|,\|\cdot\|$, and $\operatorname{tr}(\cdot)$ denote transpose, conjugate, conjugate transpose, determinant, Frobenius norm, and trace of a matrix, respectively.

\section{System model}

We consider a FD massive MIMO SWIPT system that the BS simultaneously serves a set of downlink users (cellular 
users) $\mathbf{K}_{D}=\left\{1,2, \cdots, K_{d}\right\}$ and a set of fixed uplink sensors $\mathbf{K}_{U}=\left\{1,2, \cdots, K_{u}\right\}$ which are uniformly distributed in the coverage area of the BS, as shown in Fig. 1. The number of downlink users is $K_{d}$, and the number of sensors is $K_{u}$. The BS is equipped with a separate massive antenna array. The total number of antennas at the BS is $2 N$ of which $N$ antennas are dedicated to the downlink and $N$ antennas are used for the uplink. We assume that all users and sensors are work in HD mode and have a single antenna. Each sensor employs a rectanna for $\mathrm{EH}$ and uses the harvested energy to power its subsequent data transmission.

We consider frame-based transmissions over Rayleigh fading channels. The length of one frame is fixed to $T$ seconds, which is assumed to be less than the coherence interval of the channel. We use $\mathbf{h}_{k_{u}} \in \mathbb{C}^{N \times 1}$ and $\mathbf{h}_{k_{d}} \in \mathbb{C}^{N \times 1}$ to denote the uplink UE-BS channel vector from the user $k_{u}$ to the receive antenna array of BS and the downlink UE-BS channel vector from the transmit antenna array of BS to the user $k_{d}$. Let $\mathbf{h}_{k_{u}}^{e} \in \mathbb{C}^{N \times 1}$ and $\mathbf{h}_{k_{d}}^{e} \in \mathbb{C}^{N \times 1}$ denote the uplink sensor-BS channel vector from the sensor node $k_{d}$ to the receive antenna array of the BS and the downlink sensor-BS channel vector from the transmit antenna array of the BS to the sensor node $k_{d}$. We use $\mathbf{H}_{S I} \in \mathbb{C}^{N \times N}$ to denote the SI channel matrix from the transmit antenna array to the receive antenna array of the BS.

Note that the channel model between the user and the BS is the same as the channel model between the sensor and the BS; in the following sections, we only introduce the uplink and downlink channel model between the user and the BS.

\subsection{Uplink/downlink channel model}

We consider the general cluster-based channel model [53] where the received signal at the BS from the uplink user $k_{u}$ is a sum of the contributions from $M_{u}$ scattering clusters and the received signal at user $k_{u}$ is a sum of the contributions from $M_{u}$ scattering clusters. The direction of arrival (DOA) of signals resulting from the $i$ th cluster to the BS is within the region $\left[\theta_{k_{u, i}}^{\min }, \theta_{k_{u, i}}^{\max }\right]$ and the direction of departure (DOD) region of signals resulting from the $i$ th scattering cluster to user $k_{d}$ is within the region $\left[\theta_{k_{d, i}}^{\min }, \theta_{k_{d, i}}^{\max }\right]$. Thus, the channel vector between the user $k_{u}$ or $k_{d}$ and the BS can be expressed as [53]

$$
\mathbf{h}_{k \xi}=\sum_{i=1}^{M_{\xi}} \int_{\theta_{k_{\xi, i}}^{\min }}^{\theta_{k_{\xi, i}}^{\max }} \mathbf{a}(\theta) r_{k_{\xi, i}}(\theta) d \theta
$$

where $\xi \in\{u, d\}, \mathbf{a}(\theta)=\left[1, \exp \left(-j \frac{2 \pi d \sin (\theta)}{\lambda}\right), \cdots\right.$, $\left.\exp \left(-j \frac{2 \pi d(N-1) \sin (\theta)}{\lambda}\right)\right]^{T}$ is the array response vector with $d$ and $\lambda$ denoting the antenna spacing and carrier wavelength, respectively. $r_{k_{\xi}, i}(\theta)$ denotes the complexvalued response gain of uplink/downlink channel.

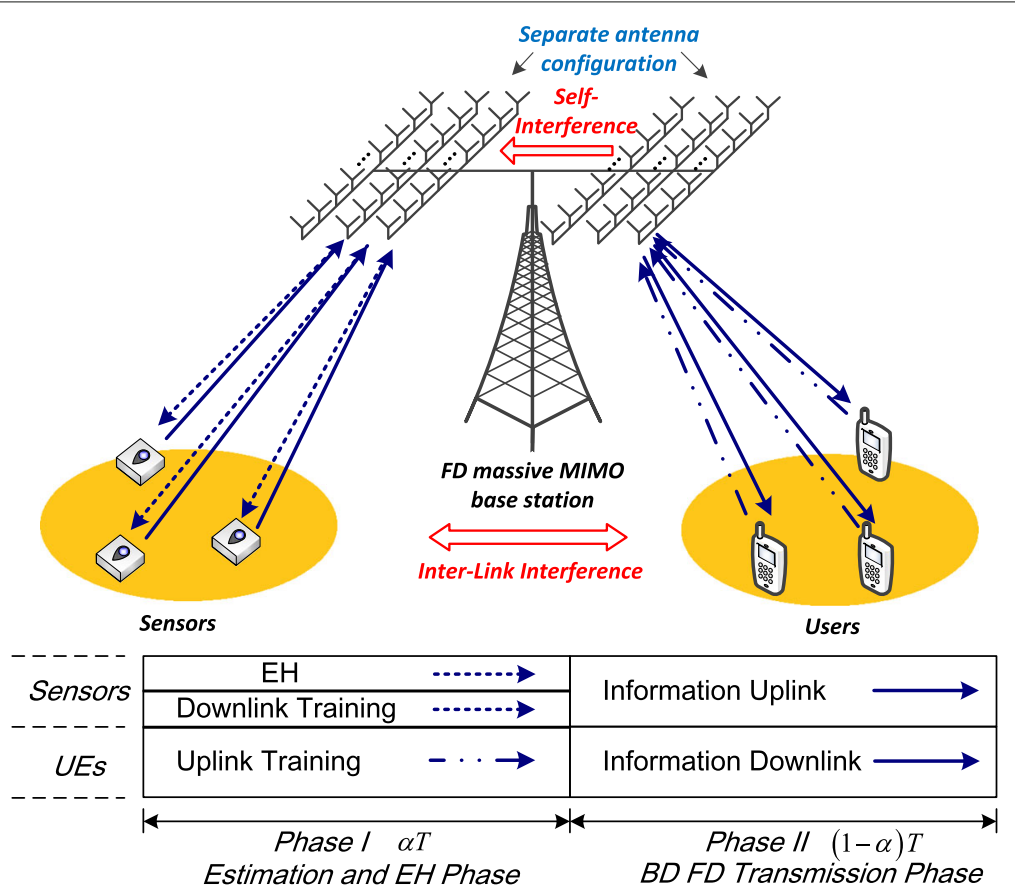

Fig. 1 Illustration of full-duplex massive MIMO SWIPT system and data frame structure 


\subsection{SI channel model}

The SI signal can be viewed as the contributions of signals from $M_{\mathrm{SI}}$ scattering clusters with different DOA and DOD regions. Thus, the SI channel matrix $\mathbf{H}_{\mathrm{SI}}$ can be expressed as

$\mathbf{H}_{\mathrm{SI}}=\sum_{i=1}^{M_{\mathrm{SI}}} \int_{\theta_{R, i}^{\min }}^{\theta_{R, i}^{\max }} \int_{\theta_{T, i}^{\max }}^{\theta_{T, i}^{\max }} r_{\mathrm{SI}, \mathrm{i}}\left(\theta_{R}, \theta_{T}\right) \mathbf{a}\left(\theta_{R}\right) \mathbf{a}^{H}\left(\theta_{T}\right) d \theta_{R} d \theta_{T}$

where $r_{\mathrm{SI}, \mathrm{i}}\left(\theta_{R}, \theta_{T}\right)$ denotes the complex-valued response gain.

In (1) and (2), we assume that the complex-valued response gains with different incidence angles are uncorrelated [54], that is

$$
\begin{aligned}
\mathbb{E}\left[r_{k_{u}, i}(\theta) r_{k_{u}, i}^{*}\left(\theta^{\prime}\right)\right] & =S_{k_{u}, i}(\theta) \delta\left(\theta-\theta^{\prime}\right) \\
\mathbb{E}\left[r_{k_{d}, i}(\theta) r_{k_{d}, i}^{*}\left(\theta^{\prime}\right)\right] & =S_{k_{d}, i}(\theta) \delta\left(\theta-\theta^{\prime}\right) \\
\mathbb{E}\left[r_{\mathrm{SI}, \mathrm{i}}\left(\theta_{R}, \theta_{T}\right) r_{\mathrm{S}, \mathrm{i}}^{*}\left(\theta_{R}^{\prime}, \theta_{T}^{\prime}\right)\right] & =S_{\mathrm{SI}, \mathrm{i}}(\theta) \delta\left(\theta_{R}-\theta_{R}^{\prime}\right) \delta\left(\theta_{T}-\theta_{T}^{\prime}\right)
\end{aligned}
$$

where $S_{\omega, i}(\cdot), \omega \in\left\{k_{u}, k_{d}, S I\right\}$, represents the product of the large-scale fading and channel power angle spectrum (PAS).

Note that the above studied channel model can be transformed into several well-known massive MIMO channels. For example, by setting $M_{u}=M_{d}=1$, we can get the "one ring" channel model introduced in [7]. The one ring model is typically used in the macro-cell environment where the uplink/downlink received signals are scattered by the vicinity of the users [53]. Moreover, by setting

$$
\begin{aligned}
r_{k_{u}, i}(\theta) & =\sum_{j} r_{k_{u}, i, j} \delta\left(\theta-\theta_{j}\right) \\
r_{k_{d}, i}(\theta) & =\sum_{j} r_{k_{d}, i, j} \delta\left(\theta-\theta_{j}\right) \\
r_{\mathrm{SI}, \mathrm{i}}\left(\theta_{R}, \theta_{T}\right) & =\sum_{j, l} r_{k_{\mathrm{SI}}, i, j, l} \delta\left(\theta_{R}-\theta_{R, j}\right) \delta\left(\theta_{T}-\theta_{T, l}\right)
\end{aligned}
$$

we obtain the ray-cluster-based spatial channel model, which is usually used for millimeter wave massive MIMO channel [55].

\section{Beam-domain channel representation}

In order to reduce the number of dimensions of the channel to be estimated as well as the amount of feedback, we resort to the BEM [56]. Using the BEM, the BD channel representation can be obtained by projecting the channel vector (matrix) on common bases. In this way, the channel can be compressed in the BD under certain selected basis spaces and the channel dimension required to be estimated can be reduced greatly. Moreover, by exploiting the BD structure of SI channel, it is possible to eliminate the SI without using the instantaneous
SI channel knowledge and hence realize efficient SWIPT transmission.

Under the BEM, the uplink/downlink UE-BS channel vector $\mathbf{h}_{k_{\xi}}, \xi \in\{u, d\}$ can be expanded from a set of uniform basis vectors $\left\{\mathbf{f}_{1}, \mathbf{f}_{2}, \cdots, \mathbf{f}_{N}\right\} \in \mathbb{C}^{N \times 1}$, that is

$$
\mathbf{h}_{k_{\xi}}=\sum_{m=1}^{N} \tilde{h}_{k_{\xi}, m} \mathbf{f}_{m}=\tilde{F}_{k_{\xi}}
$$

where $\mathbf{F}=\left[\mathbf{f}_{1}, \mathbf{f}_{2}, \cdots, \mathbf{f}_{N}\right]$. In this paper, the basis vector $\mathbf{f}_{i}$ is also called a beam [8], and $\tilde{\mathbf{h}}_{k_{\xi}}=\left[\tilde{h}_{k_{\xi}, 1}, \tilde{h}_{k_{\xi}, 2}, \cdots, \tilde{h}_{k_{\xi}, N}\right]$ is called the $\mathrm{BD}$ channel vector, which is obtained by projecting the channel vector $\mathbf{h}_{k \xi}$ onto the set of all the beams $\Gamma=\left\{\mathbf{f}_{1}, \mathbf{f}_{2}, \cdots, \mathbf{f}_{N}\right\}$. According to (1) and (5), we can obtain

$$
\tilde{\mathbf{h}}_{k_{\xi}}=\mathbf{F}^{H} \mathbf{h}_{k_{\xi}}=\sum_{i=1}^{M_{\xi}} \int_{\theta_{k_{\xi}, i}}^{\theta_{k_{\xi}, i}^{\max }} \mathbf{F}^{H} \mathbf{a}(\theta) r_{k_{\xi}, i}(\theta) d \theta
$$

By projecting channel vectors onto certain selected basis spaces, we can approximate the channel vector from user $k_{\xi}$ to $\mathrm{BS}$ as [52]

$$
\mathbf{h}_{k_{\xi}} \approx \sum_{m \in B_{k_{\xi}}} \tilde{h}_{k_{\xi}, m} \mathbf{f}_{m}=\mathbf{F}^{\left\{B_{k_{\xi}}\right\}} \widetilde{\mathbf{h}}_{k_{\xi}}^{\left\{B_{k_{\xi}}\right\}}
$$

where $B_{k_{\xi}}$ is the selected active beam set (ABS) of uplink/downlink which contains the indexes of beams with non-negligible BD channel gains. All the elements selected in the set $B_{k_{\xi}}$ is related to the DOA/DOD, and the detailed method for determining all the elements in the set of $B_{k_{\xi}}$ will be described in the following section. $\mathbf{F}^{\left\{B_{k_{\xi}}\right\}}$ is the corresponding active beam spaces, whose columns are consisted of the beams in $B_{k_{\xi}}$. The reduced-dimension BD channel vector $\left.\widetilde{\mathbf{h}}_{k_{\xi}}^{\left\{B_{k_{\xi}}\right\}} \in \mathbb{C}\right|^{B_{k_{\xi}} \mid \times 1}$ is called the BDEC. Note that (7) holds with equality as $N \rightarrow \infty$.

Based on (1) and (7), the BDEC vector can be expressed as

$$
\begin{aligned}
\widetilde{\mathbf{h}}_{k_{\xi}}^{\left\{B_{k_{\xi}}\right\}} & =\left(\mathbf{F}^{\left\{B_{k_{\xi}}\right\}}\right)^{H} \mathbf{h}_{k_{\xi}} \\
& =\sum_{i=1}^{M_{\xi}} \int_{\theta_{k_{\xi}, i}^{\min }}^{\theta_{k_{\xi}, i}^{\max }}\left(\mathbf{F}^{\left\{B_{k_{\xi}}\right\}}\right)^{H} \mathbf{a}(\theta) r_{k_{\xi}, i}(\theta) d \theta
\end{aligned}
$$

From (8), the original channel vector can be recovered from the BDEC vector if the DOA/DOD (and hence the $\mathrm{ABS}$ ) information is known. As a result, in order to obtain $\mathbf{h}_{k_{\xi}}$, it is enough to estimate the $\left|B_{k_{\xi}}\right|$-dimension BDEC during the training phase. Hence, the consumption of training resources will be significantly reduced. The ABSs $B_{k_{\xi}}, \xi \in\{d, u\}$ can be designed by solving the following problem 


$$
\begin{aligned}
& \min _{B_{k_{\xi}}}\left|B_{k_{\xi}}\right|
\end{aligned}
$$

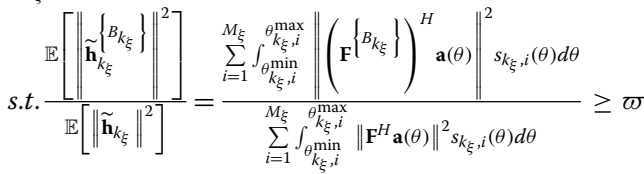

where $\varpi<1$ is the threshold.

In the same way, we can decompose the SI channel in the BD form, that is $\tilde{\mathbf{H}}_{S I}=\mathbf{F}^{H} \mathbf{H}_{S I} \mathbf{F}$. Since the average $\mathrm{BD}$ SI channel gain has non-negligible value when the beam angle of receive side $\mathbf{f}_{m}$ lies in $\cup_{i=1}^{M_{S I}}\left(\sin \theta_{R, i}^{\min }-\varepsilon, \sin \theta_{R, i}^{\max }+\varepsilon\right)$, and the beam angle of transmit side $\mathbf{f}_{n}$ lies in $\cup_{i=1}^{M_{S I}}\left(\sin \theta_{T, i}^{\min }-\varepsilon, \sin \theta_{T, i}^{\max }+\varepsilon\right)$, where $\varepsilon$ approaches to zero in the large $N$ regime. Hence, the average $\mathrm{BD}$ SI channel gain $\mathbb{E}\left[\left|\left[\tilde{\mathbf{H}}_{S I}\right]_{m, n}\right|^{2}\right]=$ $\mathbb{E}\left[\left|\mathbf{f}_{m}^{H} \mathbf{H}_{S I} \mathbf{f}_{n}\right|^{2}\right] \cdot \mathbf{x}$

Let $B_{\mathrm{SI}, \mathrm{R}}$ and $B_{\mathrm{SI}, \mathrm{T}}$ denote the ABS of the SI channel at the receive side and transmit side, respectively. $B_{\mathrm{SI}, \mathrm{R}}$ and $B_{\mathrm{SI}, \mathrm{T}}$ can be determined by solving problem in (10) on the top of this page.

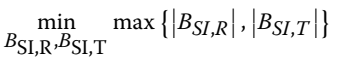

$$
\begin{aligned}
& \text { s.t. } \\
& \frac{\mathbb{E}\left[\| \tilde{\mathbf{H}}_{\mathrm{SI}}^{\left.\left.B_{\mathrm{SI}, \mathrm{R}}, B_{\mathrm{SI}, \mathrm{T}}\right\} \|^{2}\right]}\right.}{\mathbb{E}\left[\left\|\tilde{\mathbf{H}}_{\mathrm{SI}}\right\|^{2}\right]}
\end{aligned}
$$

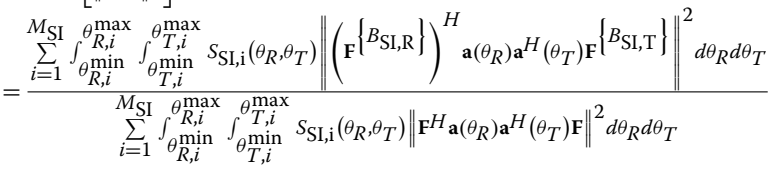

$$
\begin{aligned}
& \geq \varpi
\end{aligned}
$$

Hence, we can approximately express the BD SI chan-

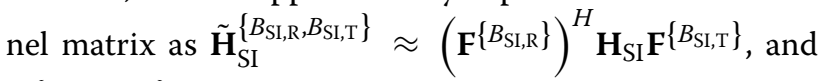
$\tilde{\mathbf{H}}_{\mathrm{SI}}^{\left\{B_{\mathrm{SI}, \mathrm{R}}, B_{\mathrm{SI}, \mathrm{T}}\right\}} \in \mathbb{C}^{\left|B_{\mathrm{SI}, \mathrm{R}}\right| \times\left|B_{\mathrm{SI}, \mathrm{T}}\right|}$ can be expressed as

$$
\begin{aligned}
\tilde{\mathbf{H}}_{\mathrm{SI}}^{\left\{B_{\mathrm{SI}, \mathrm{R}}, B_{\mathrm{SI}, \mathrm{T}}\right\}}= & \left(\mathbf{F}^{\left\{B_{\mathrm{SI}, \mathrm{R}}\right\}}\right)^{H} \mathbf{H}_{\mathrm{SI}} \mathbf{F}^{\left\{B_{\mathrm{SI}, \mathrm{T}}\right\}} \\
= & \sum_{i=1}^{M_{\mathrm{SI}}} \int_{\theta_{R, i}^{\min }}^{\theta_{R, i}^{\max }} \int_{\theta_{T, i}^{\min }}^{\theta_{T, i}^{\max }} r_{\mathrm{SI}, \mathrm{i}}\left(\theta_{R}, \theta_{T}\right)\left(\mathbf{F}^{\left\{B_{\mathrm{SI}, \mathrm{R}}\right\}}\right)^{H} \\
& \times \mathbf{a}\left(\theta_{R}\right) \mathbf{a}^{H}\left(\theta_{T}\right) \mathbf{F}^{\left\{B_{\mathrm{SI}, \mathrm{T}}\right\}} d \theta_{R} d \theta_{T}
\end{aligned}
$$

\section{BD hybrid TS and PS SWIPT protocol}

In this section, we propose a BD scheme to realize SWIPT in the cellular system with FD massive MIMO BS. In order to accomplish the channel estimation, the transmit and receive antenna arrays of the $\mathrm{BS}$ are assumed to be shared antennas, i.e., the transmit and receive RF chains share the same antenna [10]. In the frame-based BD massive MIMO FD SWIPT system, each frame is divided into two phases, phase I: estimation and EH phase and phase II: BD FD transmission phase, as shown in Fig. 1.

In the phase $I$ of time period $\alpha T(0 \leq \alpha \leq 1)$, users transmit pilots for channel estimation at the BS, while the BS simultaneously transmits energy signals to the sensor nodes for EH and channel estimation. Due to the separate antenna configuration at the BS; therefore, the reciprocity of uplink and downlink channels does not hold. The BS uses the transmit antenna array to receive the pilot signals of users for uplink UE-BS channel estimation and uses the receive antenna array to transmit the energy signals. We assume that the sensor opens the receive circuit during the interval $\alpha T$, and part of the received energy signal is used for channel estimation and part of the received energy signal is used for $\mathrm{EH}$; hence, the sensors can obtain the uplink sensor-BS channel. Specifically, the BS uses the transmit antenna array to receive the pilot signals of UEs, that is $y_{t}^{U}$ as shown in Fig. 2. Thus, the uplink UE-BS channel can be estimated during phase I. Simultaneously, the $\mathrm{BS}$ uses receive antenna array to transmit the energy signals to all the sensors. The signal received by sensor node can be expressed as $y_{r}^{S}$. Part of the received energy signal, $\sqrt{\beta} y_{r}^{S}$, is used for $\mathrm{EH}$ and part of the received signal, $\sqrt{1-\beta} y_{r}^{S}$, is used for downlink sensor-BS channel estimation, where $\beta$ denotes the PS ratio. Note that the detailed training signals (pilot signals and energy signals) design will illustrated in the next subsection.

In the phase II of time period $(1-\alpha) T$, the BS obtains the downlink UE-BS CSI based on channel reciprocity and then forms the beams for information transmission to the users. Moreover, the sensors feedback the uplink sensor-BS CSI to the BS and transmit their data during the interval $(1-\alpha) T$ using the harvested energy. Specifically, the sensors transmit signal $\sqrt{\beta \eta} y_{t}^{S}$ to the BS, where $\eta<1$ denotes the energy conversion efficiency. Note that the resource consumption for CSI feedback of sensors are omitted in this paper.

\subsection{Training design}

Let $\Phi_{u} \in \mathbb{C}^{\tau_{u} \times \max _{g_{u} \in G_{u}} K_{g_{u}}}$ be the orthogonal pilot sequence set for uplink UE-BS training, where $\tau_{u}$ denotes the length of pilot sequence which satisfies $\tau_{u} \geq \max _{g_{u} \in G_{u}} K_{g_{u}}, g_{u}$ denotes the user group, $K_{g_{u}}$ is the number of users in the group $g_{u}$, and $G_{u}$ denotes the set of user groups. The pilot sequences allocated for group $g_{u}$ can be given by $\Phi_{g_{u}}=\Phi_{u}^{\left\{: 1,1 ; K_{g_{u}}\right\}}$ and $\left|\left[\Phi_{g_{u}}\right]_{i, j}\right|^{2}=p_{u}$, where $p_{u}$ denotes the power of each uplink pilot symbol. In this paper, we consider the fixed sensors, hence it is reasonable to assume that the 


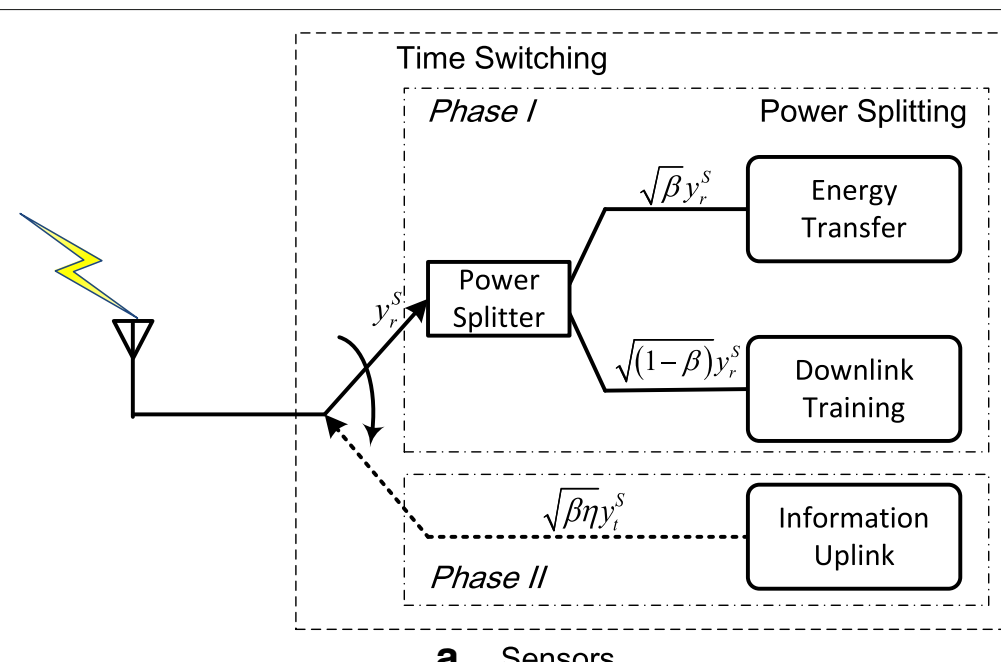

a Sensors

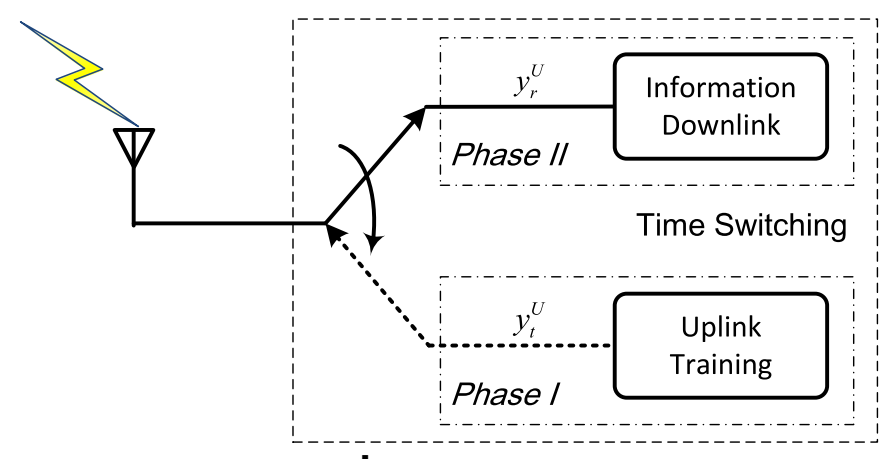

b Users

Fig. 2 Illustration of the signal flow for the proposed beam-domain hybrid TS and PS SWIPT protocol

downlink sensors group $g_{d}^{e}$ and the active beam sets $B_{g_{d}^{e}}$ are known. Let $\left|B_{g_{d}^{e}}\right|=b_{d}^{e}$ and $\Phi_{g_{d}}^{\mathrm{E}} \in \mathbb{C}^{\tau_{d} \times b_{d}^{e}}$ be the orthogonal energy sequence set, where $\tau_{d}$ denotes the length of energy sequence which satisfies $\tau_{d} \geq b_{d}^{e}$. Let $K_{g_{d}^{e}}$ denotes the number of sensors in the group $g_{d}^{e}$, and $G_{d}^{e}$ denotes the set of downlink sensor groups.

If $\tau_{d} \neq \tau_{u}$, we define

$$
\begin{cases}\dot{\Phi}_{g_{d}}^{\mathrm{E}}=\Phi_{g_{d}}^{\mathrm{E}\left\{1: \tau_{u}\right\}} & \text { if } \tau_{d} \geq \tau_{u} \\
\dot{\Phi}_{g_{d}}^{\mathrm{E}}=\left[\begin{array}{l}
\Phi_{g_{d}}^{\mathrm{E}} \\
0 \\
{ }^{\left(\tau_{u-\tau_{d}}\right) \times b_{d}}
\end{array}\right] & \text { if } \tau_{d}<\tau_{u}\end{cases}
$$

and $\left|\left[\Phi_{g_{d}}^{\mathrm{E}}\right]_{i, j}\right|^{2}=p_{d}^{e}$, the received signal at the BS is given by

$$
\mathbf{Y}_{u}=\sum_{g_{u} \in G_{u}} \mathbf{H}_{g_{u}} \Phi_{g_{u}}^{T}+\mathbf{H}_{S I} \sum_{g_{d}^{e} \in G_{d}^{e}} \mathbf{F}^{\left\{B_{g_{d}^{e}}\right\}}\left(\dot{\Phi}_{g_{d}}^{\mathrm{E}}\right)^{T}+\mathbf{N}_{u}
$$

where $\mathbf{H}_{g_{u}}=\left[\mathbf{h}_{g_{u, 1}}, \cdots, \mathbf{h}_{g_{u, K} g_{u}}\right]$ denotes the channel matrix from the user group $g_{u}$ to the BS, $\mathbf{N}_{u}$ denotes the
AWGN with variance $\sigma$. The received signal at the sensor group $g_{d}^{e}$ can be expressed as

$$
\mathbf{Y}_{g_{d}^{e}}=\mathbf{H}_{g_{d}^{e}}^{H} \sum_{g_{d}^{e} \in G_{d}^{e}} \mathbf{F}^{\left\{B_{g_{d}^{e}}\right\}}\left(\Phi_{g_{d}}^{\mathrm{E}}\right)^{T}+\mathbf{N}_{g_{d}^{e}}
$$

where $\mathbf{H}_{g_{d}^{e}}=\left[\mathbf{h}_{g_{d}^{e}, 1}, \cdots, \mathbf{h}_{g_{d}^{e}, K_{g_{d}^{e}}}\right]$ and $\mathbf{N}_{g_{d}^{e}}$ denotes the AWGN with variance $\sigma$.

\subsection{UE-BS BDEC estimation}

By multiplying both sides of (13) with $\left(\mathbf{F}^{\left\{B_{g_{u}}\right\}}\right)^{H}$, we arrive at the $\mathrm{BD}$ receive pilot signal from user group $g_{u}$

$$
\begin{aligned}
\mathbf{Y}_{g_{u}}= & \left(\mathbf{F}^{\left\{B_{g_{u}}\right\}}\right)^{H} \mathbf{Y}_{u} \\
= & \tilde{\mathbf{H}}_{g_{u}}^{\left\{B_{g_{u}}\right\}} \Phi_{g_{u}}^{T}+\sum_{g_{u}^{\prime} \in G_{u} /\left\{g_{u}\right\}} \tilde{\mathbf{H}}_{g_{u}^{\prime}}^{\left.B_{g_{u}}\right\}} \Phi_{g_{u}^{\prime}}^{T} \\
& +\sum_{g_{d}^{e} \in G_{d}^{e}} \tilde{\mathbf{H}}_{S I}^{\left\{B_{g_{u}}, B_{g_{d}^{e}}^{e}\right\}}\left(\dot{\Phi}_{g_{d}}^{\mathrm{E}}\right)^{T}+\left(\mathbf{F}^{\left\{B_{g_{u}}\right\}}\right)^{H} \mathbf{N}_{u}
\end{aligned}
$$


where $\tilde{\mathbf{H}}_{S I}^{\left\{B_{g_{u}}, B_{g_{d}^{e}}\right\}}=\left(\mathbf{F}^{\left\{B_{g_{u}}\right\}}\right)^{H} \mathbf{H}_{S I} \mathbf{F}^{\left\{B_{d}^{e}\right\}}$. With (15), the least squares (LS) estimator of the BDEC vector for the user $g_{u, k}$ within group $g_{u}$ can be obtained as

$$
\begin{aligned}
\widetilde{\mathbf{h}}_{g_{u, k}, L S}^{\left\{B_{g_{u}}\right\}}= & \frac{1}{\tau_{u} p_{u}} \mathbf{Y}_{g_{u}} \Phi_{u}^{*} \mathbf{e}_{k} \\
= & \widetilde{\mathbf{h}}_{g_{u, k}}^{\left\{B_{g_{u}}\right\}}+\sum_{g_{u}^{\prime} \in G_{u} /\left\{g_{u}\right\}} \widetilde{\mathbf{h}}_{g_{u, k}^{\prime}}^{\left\{B_{g_{u}}\right\}} \\
& +\frac{1}{\tau_{u} p_{u}} \sum_{g_{d}^{e} \in G_{d}^{e}} \tilde{\mathbf{H}}_{S I}^{\left\{B_{g_{u}}, B_{g_{d}^{e}}\right\}}\left(\dot{\Phi}_{g_{d}}^{\mathrm{E}}\right)^{T} \Phi_{u}^{*} \mathbf{e}_{k} \\
& +\frac{1}{\tau_{u} p_{u}}\left(\mathbf{F}^{\left\{B_{g_{u}}\right\}}\right)^{H} \mathbf{N}_{u} \Phi_{u}^{*} \mathbf{e}_{k}
\end{aligned}
$$

where $p_{u}$ denotes the power of each pilot symbol transmitted by users. The second term of right-hand sides of (16) indicates the pilot contamination due to the use of the same pilot sequence set over all the user groups. The third term is the SI due to the simultaneous uplink training and downlink energy transmission.

In the practical system with finite number of BS antennas, the linear minimum mean square error (LMMSE) estimator can be used to refine the results of the LS estimator in (16) to mitigate the residual pilot contamination and SI. Based on the general expression of LMMSE estimator, the refined estimates can be expressed as

$$
\begin{aligned}
& \widetilde{\mathbf{h}}_{g_{u, k}, L M}^{\left\{B_{g_{u}}\right\}}=\sum_{i=1}^{M_{u}} \int_{\theta_{g_{u, k, i}}^{\min , i}}^{\theta_{g_{u}}^{\max }}\left(\mathbf{F}^{\left\{B_{g_{u}}\right\}}\right)^{H} \mathbf{a}(\theta) \mathbf{a}^{H}(\theta) \mathbf{F}^{\left\{B_{g_{u}}\right\}_{g_{u, k, i}}} S_{g}(\theta) d \theta \\
& \times\left(\sum_{g_{u}^{\prime} \in G_{u}} \sum_{i=1}^{M_{u}} \int_{\theta_{g_{u, k, i}^{\prime}}^{\min }}^{\theta_{g^{\prime}, k, i}^{\max }}\left(\mathbf{F}^{\left\{B_{g_{u}}\right\}}\right)^{H} \mathbf{a}(\theta) \mathbf{a}^{H}(\theta) \mathbf{F}^{\left\{B_{g_{u}}\right\}} S_{g_{u^{\prime}, k, i}}(\theta) d \theta\right. \\
& +\left(\frac{1}{\tau_{u} p_{u}}\right)^{2} \sum_{B_{g_{d}^{e}}, B_{g_{d}^{\prime}} \in G_{d}^{e}} \sum_{i=1}^{M_{S I}} \int_{\theta_{R, i}^{\min }}^{\theta_{R_{R}}^{\max }} \int_{\theta_{T, i}^{\min }}^{\theta_{T, i}^{\max }} \mathbf{G}_{\theta_{R}, \theta_{T}}^{\left\{B_{g_{u}}, B_{g_{d}^{e}}\right\}} \Psi_{k} \\
& \left.\times\left(\mathbf{G}_{\theta_{R}, \theta_{T}}^{\left\{B_{g_{u}}, B_{g^{\prime}}\right\}}\right)^{H} S_{S I, i}\left(\theta_{R}, \theta_{T}\right) d \theta_{R} d \theta_{T}+\frac{\sigma}{\tau_{u} p_{u}} \mathbf{I}_{b_{u}}\right)^{-1} \widetilde{\mathbf{h}}_{g_{u, k}, L S}^{\left\{B_{g_{u}}\right\}}
\end{aligned}
$$

where $\left.\mathbf{G}_{\theta_{R}, \theta_{T}}^{\left\{B_{g_{u}}, B_{g^{\prime}}\right.}\right\} \triangleq\left(\mathbf{F}^{\left\{B_{g_{u}}\right\}}\right)^{H} \mathbf{a}\left(\theta_{R}\right) \mathbf{a}^{H}\left(\theta_{T}\right) \mathbf{F}^{\left\{B_{g^{\prime}} e_{d}\right\}}$ and meanwhile $\Psi_{k}=\left(\dot{\Phi}_{g_{d}}^{\mathrm{E}}\right)^{T} \Phi_{u}^{*} \mathbf{e}_{k} \mathbf{e}_{k}^{H} \Phi_{u}^{T}\left(\dot{\Phi}_{g_{d}}^{\mathrm{E}}\right)^{*}$. The BSto-users channel CSI $\widetilde{\mathbf{h}}_{g_{d, k}, L M}^{\left\{B_{g_{d}}\right\}}$ is obtained by using the reciprocity properties of the wireless channel.

\subsection{Energy transfer for sensors}

During phase $I$, the received signal at the $g_{d, k}^{e}$ th sensor can be expressed by

$\mathbf{y}_{g_{d, k}^{e}}^{e}=\mathbf{h}_{g_{d, k}^{e}}^{H} \sum_{g_{d}^{\prime e} \in G_{d}^{e}} \mathbf{F}^{\left\{B_{g^{\prime}}^{e}\right\}}\left(\Phi_{g_{d}}^{\mathrm{E}}\right)^{T}+\sum_{k_{u}=1}^{K_{d}} h_{k_{u}, g_{d, k}^{e}} \Phi_{k_{u}}^{T}+\mathbf{n}_{g_{d, k}^{e}}$ where $h_{k_{u}, g_{d, k}^{e}} \in \mathbb{C}^{1 \times 1}$ denotes the inter-user interference (IUI) channel between the $k_{u}$ th user and the $g_{d, k}^{e}$ th sensor node, $\Phi_{k_{u}}$ is one row of $\Phi_{u}$ and denotes the pilot sequence transmit by user $k_{u}$, and $\mathbf{n}_{g_{d, k}^{e}} \sim \mathcal{C N}\left(0, \sigma^{2}\right)$ denotes the AWGN at the $g_{d, k}^{e}$ th sensor node.

Generally, the EH receiver will harvest energy from part of the whole signal, that is $\sqrt{\beta} \mathbf{y}_{d, k}^{e}$. However, the noise is negligible compared with the high-power signal and is thereby omitted in the harvested energy [39]. We further assume that the amount of energy harvested from the cellular users' transmissions is negligible due to their low transmit power. Therefore, the $g_{d, k}^{e}$ th sensor node transmit power during the remaining $(1-\alpha) T$ time can be written as

$$
\begin{aligned}
p_{g_{d, k}^{e}}^{\mathrm{H}} & \left.=\frac{\eta \beta}{(1-\alpha) T} \| \mathbf{h}_{g_{d, k}^{e}}^{\mathrm{H}} \sum_{g_{d}^{\prime e} \in G_{d}^{e}} \mathbf{F}^{B_{g_{d}^{\prime}}^{e}}\right\}\left(\Phi_{g_{d}}^{\mathrm{E}}\right)^{T} \|^{2} \\
& \approx \frac{\eta \beta}{(1-\alpha) T}\left\|\mathbf{h}_{g_{d, k}^{e}}^{\mathrm{H}} \mathbf{F}^{\left\{B_{g_{d}^{e}}\right\}}\left(\Phi_{g_{d}}^{\mathrm{E}}\right)^{T}\right\|^{2} \\
& =\frac{\eta \beta}{(1-\alpha) T}\left\|\left(\tilde{\mathbf{h}}_{g_{d, k}^{e}}^{\left\{B_{g_{d}^{e}}\right\}}\right)^{H}\left(\Phi_{g_{d}}^{\mathrm{E}}\right)^{T}\right\|^{2} \\
& =\frac{\eta \beta}{(1-\alpha) T} \tau_{d} p_{g_{d}^{e}}\left\|\tilde{\mathbf{h}}_{g_{d, k}^{e}}^{\left\{B_{g_{d}^{e}}\right\}}\right\|^{2} \triangleq \frac{\alpha}{(1-\alpha)} \hat{p}_{g_{d, k}^{e}}^{\mathrm{H}}
\end{aligned}
$$

where $\|\cdot\|$ denotes the Euclidean norm of a vector, $0<$ $\eta<1$ denotes the energy conversion efficiency. $\hat{p}_{g_{d, k}^{e}}^{\mathrm{H}}=$ $p_{g_{d}^{e}} q_{g_{d, k}^{e}}$, where $\left.q_{g_{d, k}^{e}}=\eta \beta \| \tilde{\mathbf{h}}_{g_{d, k}^{e}}^{\left\{B_{g_{d}^{e}}^{e}\right.}\right\} \|^{2}$. It is worth noting that for a fixed sensor node, the downlink sensor grouping and the ABS remain unchanged. Therefore, when the instantaneous channel information is not available, effective energy transmission can be achieved only by using the information of the ABS, regardless of how the energy sequences are allocated.

The left part of the whole signal, that is $\sqrt{1-\beta} \mathbf{y}_{d, k}^{e}$, is used for the channel estimation. Since the sensor knows the values of $\beta$ and can accurate compensate it, hence we omit it in the following channel estimation. The LS channel estimator of the downlink sensor-BS channel can be expressed as

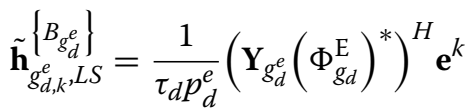

$$
\begin{aligned}
& =\tilde{\mathbf{h}}_{g_{d, k}^{e}}^{\left\{B_{g_{d}^{e}}\right\}}+\sum_{g_{d}^{\prime e} \in G_{d}^{e} / g_{d}^{e}} \tilde{\mathbf{h}}_{g_{d, k}^{e}}^{\left\{B_{g^{\prime}}\right\}}+\frac{1}{\tau_{d} p_{d}^{e}}\left(\mathbf{N}_{g_{d}^{e}}\left(\Phi_{g_{d}}^{\mathrm{E}}\right)^{*}\right)^{H} \mathbf{e}^{k}
\end{aligned}
$$


where $p_{d}^{e}$ denotes the power of each downlink energy sequence symbol, i.e., $\left|\left[\Phi_{g_{d}}^{\mathrm{E}}\right]_{i, j}\right|^{2}=p_{g_{d}^{e}}$. Similarly, we can refine the estimates with the LMMSE procedure, which resulting in

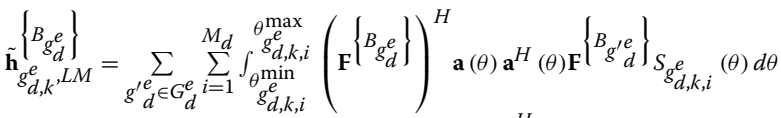

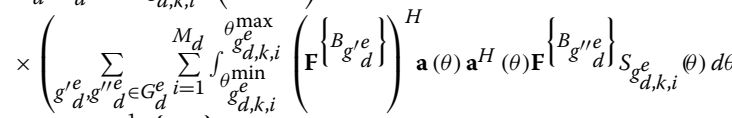

$$
\begin{aligned}
& \left.+\frac{\sigma}{\tau_{d} p^{p}}\right)^{-1} \tilde{\mathbf{h}}_{g_{d, k}^{e}}\left\{g_{d S}^{B_{e}}\right\}
\end{aligned}
$$

The uplink sensors-to-BS channel CSI $\tilde{\mathbf{h}}_{g_{u, k}^{e}, L M}^{\left\{B_{g_{u}}\right\}}$ is obtained by using the reciprocity properties of the wireless channel.

\subsection{Beam-domain FD information transmission}

The BS uses the estimated BDEC to perform beamforming to transmit information to the $K_{d}$ users. At the same time, it receives data from the $K_{u}$ sensors.

\subsubsection{BS-to-UE downlink transmission}

In downlink, the received signal at user group $g_{d}$ can be expressed as

$$
\mathbf{y}_{g_{d}}=\mathbf{H}_{g_{d}}^{H} \sum_{g_{d}^{\prime} \in G_{d}} \mathbf{x}_{g_{d}^{\prime}}+\mathbf{H}_{K_{u}, K_{d}}^{H} \mathbf{x}_{u, K_{u}}+\mathbf{n}_{g_{d}}
$$

where $\mathbf{n}_{g_{d}}$ denotes the AWGN vector with variance $\sigma$, $\mathbf{x}_{g_{d}} \in \mathbb{C}^{N \times 1}$ denotes the precoded transit signal of the BS, $\mathbf{H}_{g_{d}}=\left[\mathbf{h}_{g_{d, 1}}, \cdots, \mathbf{h}_{g_{d,} K_{g_{d}}}\right]$ denotes the channel matrix from the user group $g_{d}$ to the BS, $\mathbf{H}_{K_{u}, g_{d}} \in \mathbb{C}^{K_{u} \times K_{g_{d}}}$ denotes the channel matrix from $K_{u}$ sensors to the user group $g_{d}$, and $\mathbf{x}_{u, K_{u}} \in \mathbb{C}^{K_{u} \times 1}$ denotes the transmit signal vector of $K_{u}$ sensors.

By using the definition $\mathbf{x}_{g_{d}}=\mathbf{F}\left\{{ }^{B_{g_{d}}}\right\}_{\tilde{\mathbf{x}}_{g_{d}}}$, and substituting it into (22), we can rewrite the BD received signal at user group $g_{d}$ as

$$
\begin{aligned}
\tilde{\mathbf{y}}_{g_{d}}= & \left(\tilde{\mathbf{H}}_{g_{d}}^{\left\{B_{g_{d}}\right\}}\right)^{H} \tilde{\mathbf{x}}_{g_{d}}+\sum_{g_{d}^{\prime} \in G_{d}}\left(\tilde{\mathbf{H}}_{g_{d}}^{\left\{B_{g_{d}^{\prime}}\right\}}\right)^{H} \tilde{\mathbf{x}}_{g_{d}^{\prime}} \\
& +\mathbf{H}_{K_{u}, K_{d}}^{H} \mathbf{x}_{u, K_{u}}+\mathbf{n}_{g_{d}}
\end{aligned}
$$

where $\tilde{\mathbf{H}}_{g_{d}}^{\left\{B_{g_{d}}\right\}}=\left(\mathbf{F}^{\left\{B_{g_{d}}\right\}}\right)^{H} \mathbf{H}_{g_{d}}$. Note that the second term on the right-hand sides of (23) indicate the intergroup interferences (IGIs). Using the interference control scheme, such as cell sectorization-based uplink-downlink user scheduling [52], the interference from sensors to users, that is, the third term on the right-hand sides of (23), can be mitigated. Hence, the BD received signal at user group $g_{d}$ can be rewritten as

$$
\tilde{\mathbf{y}}_{g_{d}}=\left(\tilde{\mathbf{H}}_{g_{d}}^{\left\{B_{g_{d}}\right\}}\right)^{H} \tilde{\mathbf{x}}_{g_{d}}+\sum_{g_{d}^{\prime} \in G_{d}}\left(\tilde{\mathbf{H}}_{g_{d}}^{\left\{B_{g_{d}^{\prime}}\right\}}\right)^{H} \tilde{\mathbf{x}}_{g_{d}^{\prime}}+\mathbf{n}_{g_{d}}
$$

To mitigate the IGI and IUI, the BS employs the BD beamforming. The intended signal of user group $g_{d}$, i.e., $\mathbf{s}_{g_{d}} \in \mathbb{C}^{K_{g_{d}} \times 1}$, is precoded by the beamforming matrix $\mathbf{W}_{\mathrm{g}_{d}}=\left[\mathbf{w}_{\mathrm{g}_{d}, 1}, \mathbf{w}_{\mathrm{g}_{d}, 2}, \cdots, \mathbf{w}_{\mathrm{g}_{d}, K_{g_{d}}}\right] \in \mathbb{C}^{b_{d} \times K_{g_{d}}}$, and $b_{d}$ denotes the number of beams in the ABS $B_{g_{d}}$, that is $\left|B_{g_{d}}\right|=b_{d}$, which means that the users in the user group $g_{d}$ detect signal only on their active beam spaces. Thus, the BD transmit signal vector for user group $g_{d}$ can be expressed as $\tilde{\mathbf{x}}_{g_{d}}=\mathbf{W}_{\mathrm{g}_{d}} \mathbf{s}_{g_{d}} \in \mathbb{C}^{b_{d} \times 1}$. Using these on (24), the $\mathrm{BD}$ received signal at user $g_{d_{k}}$ can be expressed as

$$
\begin{aligned}
\tilde{y}_{g_{d, k}}= & \left(\tilde{\mathbf{h}}_{g_{d, k}}^{\left\{B_{g_{d}}\right\}}\right)^{H} \mathbf{w}_{g_{d, k}} s_{g_{d, k}}+\left(\tilde{\mathbf{h}}_{g_{d, k}}^{\left\{B_{g_{d}}\right\}}\right)^{H} \sum_{k^{\prime}=1, k^{\prime} \neq k}^{K_{g_{d}}} \mathbf{w}_{g_{d, k^{\prime}}} s_{g_{d, k^{\prime}}} \\
& +\sum_{g_{d}^{\prime} \in G_{d} /\left\{g_{d}\right\}}\left(\tilde{\mathbf{h}}_{g_{d, k}}^{\left\{B_{g_{d}^{\prime}}\right\}}\right)^{H} \mathbf{w}_{g^{\prime} d} \mathbf{s}_{g_{d}^{\prime}}+n_{g_{d, k}}
\end{aligned}
$$

The optimal beamforming scheme to maximize the sum rate has been proved NP-hard [57]. Thus, in this paper, we consider the suboptimal linear precoding scheme, i.e., ZF beamforming, to approximate the system performance upper bound. Assuming the channel estimators in (19), the transmit beamforming matrices of the BS can be expressed as

$$
\mathbf{W}_{\mathrm{g}_{d}}=\tilde{\mathbf{H}}_{g_{d, L M}}^{\left\{B_{g_{d}}\right\}}\left(\left(\tilde{\mathbf{H}}_{g_{d, L M}}^{\left\{B_{g_{d}}\right\}}\right)^{H} \tilde{\mathbf{H}}_{g_{d, L M}}^{\left.B_{g_{d}}\right\}}\right)^{-1} \Upsilon_{g_{d}}^{-1 / 2}
$$

where $\Upsilon_{g_{d}}$ is a diagonal normalized matrix with $\left[\Upsilon_{g_{d}}\right]_{l, l}$ can be expressed as $\left[\Upsilon_{g_{d}}\right]_{l, l}=$ $\mathbf{e}_{l}^{H}\left(\left(\tilde{\mathbf{H}}_{g_{d, L M}}^{\left\{B_{g_{d}}\right\}}\right)^{H} \tilde{\mathbf{H}}_{g_{d, L M}}^{\left\{B_{g_{d}}\right\}}\right)^{-1} \mathbf{e}_{l}$.

\subsubsection{Sensor-to-BS uplink transmission}

In uplink, the sensor $g_{d, k}^{e}$ transmit signal using the harvested energy $p_{g_{d, k}^{e}}^{\mathrm{H}}$. In the following, the transmit power of sensor $g_{d, k}^{e}$ is set to $p_{g_{u, k}^{e}}=p_{g_{d, k}^{e}}^{\mathrm{H}}=\left|s_{g_{u, k}^{e}}\right|^{2}$, where $s_{g_{u, k}^{e}}$ denotes the transmit signal of sensor $g_{u, k}^{e}$. The received signal at the BS can be expressed as 


$$
\mathbf{y}_{u}^{e}=\sum_{g_{u}^{\prime e} \in G_{u}} \mathbf{H}_{g^{\prime}{ }_{u}} \mathbf{S}_{g^{\prime}{ }_{u}}+\mathbf{H}_{S I} \sum_{g_{d}^{\prime} \in G_{d}} \mathbf{x}_{g_{d}^{\prime}}+\mathbf{n}_{u}^{e}
$$

where $\mathbf{n}_{u}^{e}$ denotes the AWGN vector with variance $\sigma$. $\mathbf{s}_{g_{u}^{e}} \in \mathbb{C}^{K_{g_{u}}^{e} \times 1}$ denotes the transmit signal of sensor group $g_{u}^{e} \cdot \mathbf{x}_{g_{d}} \in \mathbb{C}^{N \times 1}$ denotes the precoded transit signal of the BS. By multiplying both sides of (27) with $\mathbf{F}^{\left\{g_{u}\right\}}$, we arrive at the $\mathrm{BD}$ received signal at the $\mathrm{BS}$ from sensor group $g_{u}^{e}$.

$$
\begin{aligned}
\tilde{\mathbf{y}}_{g_{u}}^{e} & =\mathbf{F}{ }^{\left\{g_{u}\right\}} \mathbf{y}_{\mathbf{y}_{u}^{e}}^{e} \\
& =\tilde{\mathbf{H}}_{g_{u}^{e}}\left\{B_{g_{u}}\right\} \mathbf{s}_{g_{u}^{e}+}+\sum_{g^{\prime}{ }_{u} \in G_{u}^{e} /\left\{g_{u}^{e}\right\}} \tilde{\mathbf{H}}_{g^{\prime}{ }_{u}}\left\{B_{g_{u}}\right\} \mathbf{s}_{g_{g_{u}^{\prime}}+}+\sum_{g_{d}^{\prime} \in G_{d}} \tilde{\mathbf{H}}_{S I}\left\{B_{u}, B_{g_{d}^{\prime}}\right\}_{\tilde{\mathbf{x}}_{g_{d}^{\prime}}+\tilde{\mathbf{n}}_{g_{u}}}
\end{aligned}
$$

where $\tilde{\mathbf{n}}_{g_{u}^{e}}=\mathbf{F}{ }^{\left\{B_{u}\right\}} \mathbf{n}_{u}^{e}$. To detect the signals from sensor group $g_{u}^{e}$, the BS multiplies the BD received signal (28) with the receive beamforming matrix $\mathbf{W}_{g_{u}^{e}}=$ $\left[\mathbf{w}_{g_{u, 1}^{e}}, \mathbf{w}_{g_{u, 2}^{e}}^{e}, \cdots, \mathbf{w}_{g_{u}^{e}}, K_{g_{u}}^{e}\right] \in \mathbb{C}^{b_{u}^{e} \times K_{g_{u}}^{e}}$, i.e., $\dot{\mathbf{y}}_{g_{u}^{e}}=\mathbf{w}_{g_{u}^{e}}^{H} \tilde{\mathbf{y}}_{g_{u}^{e}}^{e}$. The $k$ th entry of $\dot{\mathbf{y}}_{g_{u}^{e}}$, that is $\dot{y}_{g_{u, k}^{e}}$, can be expressed as

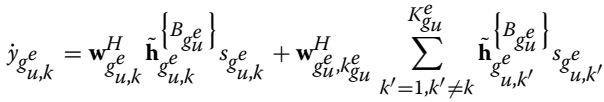

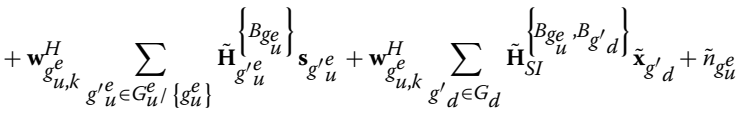

The transmit symbol of sensor $g_{u, k}^{e}$ can be recovered by using signal $\dot{y}_{g_{u, k}^{e}}$. The signal-to-interference-and-noise ratio (SINR) at the BS of sensor $g_{u, k}^{e}$ can be expressed as

$$
\operatorname{SINR}_{g_{u, k}^{e}}=\frac{p_{g_{u, k}^{e}}^{e}\left|\mathbf{w}_{g_{u, k}^{e}}^{H} \tilde{\mathbf{h}}_{g_{u, k}^{e}}^{\left\{B_{g_{u}^{e}}\right\}}\right|^{2}}{\sigma^{2}\left\|\mathbf{w}_{g_{u, k}^{e}}^{e}\right\|^{2}+\Theta_{g_{u, k}^{e}}}
$$

where $\Theta_{g_{u, k}^{e}}$ denotes the sum of powers of channel estimation error. IUI within the group and IGI can be expressed as (31), where $\Lambda_{g_{d}}$ denotes the diagonal input covariance matrix of the downlink group $g_{d} . \Lambda_{g_{u}^{e}}=\mathbb{E}\left[\mathbf{s}_{g_{u}^{e}} \mathbf{s}_{g_{u}^{e}}^{H}\right]$ denotes the diagonal input covariance matrix of the downlink group $g_{u}^{e}$.

$$
\begin{aligned}
& \Theta_{g_{u, k}^{e}}=p_{g_{u, k}^{e}}\left|\mathbf{w}_{g_{u, k}^{e}}^{H} \Delta \tilde{\mathbf{h}}_{g_{u, k}^{e}}^{\left\{B_{g_{u}^{e}}^{e}\right\}}\right|^{2}+\sum_{k^{\prime}=1, k^{\prime} \neq k}^{K_{g_{u}}^{e}} p_{g_{u, k^{\prime}}^{e}}\left|\mathbf{w}_{g_{u, k}^{e}}^{H} \Delta \tilde{\mathbf{h}}_{g_{u, k^{\prime}}^{e}}^{\left\{B_{g_{u}^{e}}^{e}\right\}}\right|^{2} \\
& +\sum_{g_{u}^{\prime e} \in G_{u} /\left\{g_{u}^{e}\right\}}\left\|\mathbf{w}_{g_{u, k}^{e}}^{H} \tilde{\mathbf{H}}_{g_{u}^{\prime}}^{\left\{B_{g_{u}^{e}}\right\}} \Lambda_{g_{u}^{\prime e}}^{1 / 2}\right\|^{2} \\
& +\sum_{g_{d}^{\prime} \in G_{d}}\left\|\mathbf{w}_{g_{u, k}^{e}}^{H} \tilde{\mathbf{H}}_{S I}^{\left\{B_{g_{u}^{e}, B_{g_{d}^{\prime}}}\right\}} \mathbf{W}_{g_{d}^{\prime}} \Lambda_{g_{d}^{\prime}}^{1 / 2}\right\|^{2}
\end{aligned}
$$

Hence, the optimum $\mathbf{w}_{g_{u, k}^{e}}$ is the one that maximizes the SINR $_{g_{u, k}}$ and, thus, is obtained by solving the following problem

$$
\max _{\left\|\mathbf{w}_{g_{u, k}^{e}}\right\|=1} \frac{p_{g_{u, k}^{e}} \mathbf{w}_{g_{u, k}^{H}}^{H} \tilde{\mathbf{h}}_{g_{u, k}^{e}}^{\left\{B_{g_{u}^{e}}\right\}}\left(\tilde{\mathbf{h}}_{g_{u, k}^{e}}^{\left\{B_{g_{u}^{e}}\right\}}\right)^{H} \mathbf{w}_{g_{u, k}^{e}}}{\mathbf{w}_{g_{u, k}^{e}}^{H}\left(\Xi_{g_{u, k}^{e}}\right) \mathbf{w}_{g_{u, k}^{e}}}
$$

where $\Xi_{g_{u, k}^{e}}$ can be expressed as

$$
\begin{aligned}
& \Xi_{g_{u, k}^{e}}=p_{g_{u, k}^{e}} \Delta \tilde{\mathbf{h}}_{g_{u, k}^{e}}^{\left\{B_{g_{u}}\right\}}\left(\Delta \tilde{\mathbf{h}}_{g_{u, k}^{e}}^{\left\{B_{u}^{e}\right\}}\right)^{H} \\
& +\sum_{k^{\prime}=1, k^{\prime} \neq k}^{K_{g_{u}^{e}}} p_{g_{u, k^{\prime}}^{e}} \Delta \mathbf{h}_{g_{u, k^{\prime}}^{e}}^{\left.B_{g_{u}^{e}}^{e}\right\}}\left\{\left(\Delta \tilde{\mathbf{h}}_{g_{u, k^{\prime}}^{e}, L M}^{\left\{B_{g_{u}^{e}}\right\}}\right)^{H}\right.
\end{aligned}
$$

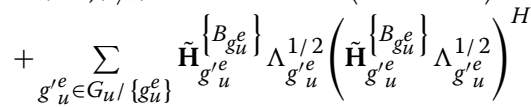

$$
\begin{aligned}
& \left.+\sum_{g_{d}^{\prime} \in G_{d}} \tilde{\mathbf{H}}_{S I}^{\left\{B_{g_{u}^{e}}^{e}, B_{g_{d}^{\prime}}\right\}} \mathbf{W}_{g_{d}^{\prime}} \Lambda_{g_{d}^{\prime}}^{1 / 2}\left(\tilde{\mathbf{H}}_{S I} B_{g_{u}^{e}}^{e, B_{g_{d}^{\prime}}}\right\} \mathbf{W}_{g_{d}^{\prime}} \Lambda_{g_{d}^{\prime}}^{1 / 2}\right)^{H}+\sigma^{2} \mathbf{I}_{b_{u}^{e}}^{e}
\end{aligned}
$$

The problem in (32) is in generalized Rayleigh quotient form. It is well known that the maximum value in (32) is obtained when $\mathbf{w}_{g_{u, k}^{e}}$ equals to the generalized eigenvector corresponding to the maximum generalized eigenvalue.

\section{Beam-domain user and sensor grouping}

The key idea of the proposed BD FD massive MIMO SWIPT scheme lies in partitioning users and sensors according to their ABSs to realize efficient energy and information transmission. In particular, we divide the users and sensors into groups under the following two criterions:

- Criterion 1: The sensors or users with the same ABS are collected in the same sensor/user group. The ABSs of different sensor/user groups are non-overlapping with certain guard interval $\mho$. Let 
$B_{g_{u}}$ and $B_{g_{u}^{\prime}}$ be the ABSs of two arbitrary user groups $g_{u}$ and $g_{u}^{\prime}$, and $B_{g_{u}}$ and $B_{g_{u}^{\prime}}$ should satisfy $\mathcal{D}\left(B_{g_{u}}, B_{g_{u}^{\prime}}\right) \geq \mho$, as shown in Fig. 3. Let $B_{g_{d}}$ and $B_{g_{d}^{\prime}}$ be the ABSs of two arbitrary sensor groups $g_{d}$ and $g_{d}^{\prime}$, and $B_{g_{d}}$ and $B_{g_{d}^{\prime}}$ should satisfy $\mathcal{D}\left(B_{g_{d}}, B_{g_{d}^{\prime}}\right) \geq \mho$.

- Criterion 2: The complete ABS of all the user groups or sensor groups is non-overlapping with the receive $\mathrm{ABS}$ or transmit $\mathrm{ABS}$ of the SI channel under certain guard interval $\mho$. Let $G_{u}$ and $G_{d}$ be the complete set of sensor groups and user groups, respectively. The ABSs $B_{g_{u}}$ and $B_{g_{d}}$ satisfy $\mathcal{D}\left(\bigcup_{g_{u} \in G_{u}} B_{g_{u}}, B_{S I, R}\right)>\mho$ or $\mathcal{D}\left(\bigcup_{g_{d} \in G_{d}} B_{g_{d}}, B_{S I, T}\right)>\mho$.

In the practical implementation, the users and sensors with different ABSs must be partitioned so that the above two conditions are satisfied as close as possible, as shown in Fig. 3. For all the users or sensors, the ABS of the SI channel at the receive side $B_{S I, R}$ and transmit side $B_{S I, T}$ should be took into consideration when carry out grouping. In the following, we proposed a BD user and sensor grouping scheme, which consists of the following two steps.

Step 1: Remove the ABS of the SI channel at the receive side $B_{S I, R}$ and transmit side $B_{S I, T}$ from the set of all the beams $\Gamma$ with a certain guard interval $\mho$.

Step2: Remove all the beams that satisfy the adjacent $\mho$ beams including itself which are not occupied by any user or sensor. We obtain serval beam sets, each of which contains several continuous beams occupied by different users or sensors. For each beam set with several continuous beams, the corresponding users or sensors form a user and sensor group.

In this way, the self-interference at the BS can be mitigated effectively based on the statistical CSI of SI channel, that is, the ABS of SI channel, rather than the instantaneous CSI of SI channel. Note that users and sensors should be grouped independently. Meanwhile, to mitigate the interference between the uplink and downlink, the user group or sensor group with overlap beams cannot be allocated with the same frequency band for transmission.

\section{Achievable rate analysis and optimization}

We carry out the achievable rate analysis and optimization in this section. In this paper, the part of the time used for channel estimation at the BS and energy harvesting $\alpha T$ can be determined by $\alpha T=\tau_{d} \geq \tau_{u}$. It is worth noting that when $\alpha T \geq \tau_{u}$, more time is allocated to the sensors for $\mathrm{EH}$ which as a result will increase the harvested energy and hence increase the transmit power of sensors. But for the users, less time is allocated for transmitting and hence reduce the SE.

\subsection{Downlink achievable rate}

\subsubsection{Downlink achievable rate with perfect CSI}

With perfect CSI, the downlink achievable rate of user $g_{d, k}$ can be expressed as

$$
\ddot{R}_{g_{d, k}}(\alpha)=(1-\alpha) \log _{2}\left(1+\ddot{\gamma}_{g_{d, k}}\right)
$$

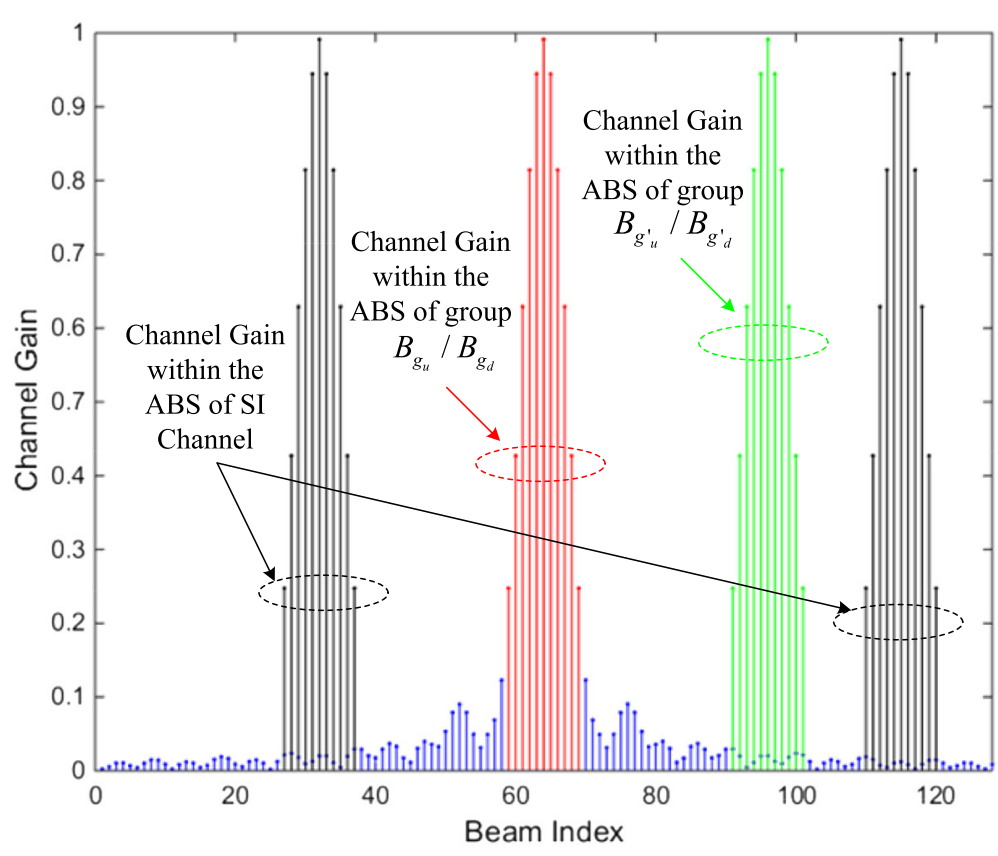

Fig. 3 Illustration of the ABSs of different sensor/user groups and the SI channel 
where $\ddot{\gamma}_{g_{d, k}}$ denotes the SINR of user $g_{d, k}$ and can be written as

$$
\ddot{\gamma}_{g_{d, k}}=\frac{p_{g_{d, k}} \mathbb{E}\left[\left[\Upsilon_{g_{d}}^{-1}\right]_{k, k}\right]}{\sum_{g_{d}^{\prime} \in G_{d} /\left\{g_{d}\right\}}\left\|\left(\tilde{\mathbf{h}}_{g_{d, k}}^{\left.B_{g_{d}^{\prime}}\right\}}\right)^{H} \mathbf{w}_{g_{d}^{\prime}} \Lambda_{g_{d}^{\prime}}\right\|^{2}+1}
$$

\subsubsection{Downlink achievable rate with imperfect CSI}

Since perfect channel estimation does not exist in real systems, hence we let $\Delta \tilde{\mathbf{h}}_{g_{d, k}, L M}^{\left\{B_{g_{d}}\right\}}=\mathbf{h}_{g_{d, k}, L M}^{\left\{B_{g_{d}}\right\}}-\tilde{\mathbf{h}}_{g_{d, k}, L M}^{\left\{B_{g_{d}}\right\}}$ denote the channel estimation error of LMMSE estimator. According to (25) and using the bounding technique in [58], the average achievable rates at user $g_{d, k}$ can be expressed as (36) where $p_{g_{d, k}}=\mathbb{E}\left[\left|s_{g_{d, k}}\right|^{2}\right]$ denotes the transmit powers. The powers of channel estimation error (CEE), IUI within the group and IGI, are explained in the equation.

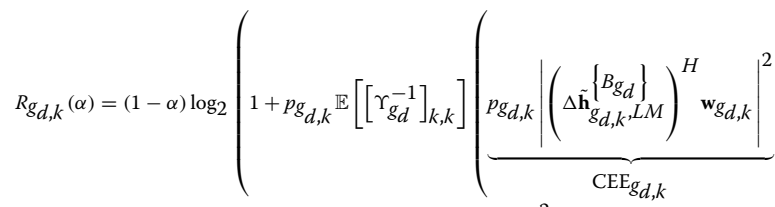

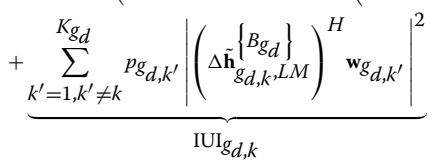

$$
\begin{aligned}
& +\underbrace{\sum_{g_{d}^{\prime} \in G_{d^{\prime}}\left\{g_{d}\right\}} \|\left(\tilde{\hat{\mathrm{g}}}_{g_{d, k}}\left[g_{g^{\prime}, d}\right\}\right.}_{\mathrm{IGI}_{g_{d, k}}})^{H} \mathbf{w}_{g_{d}^{\prime}} \Lambda_{g_{d}^{\prime}} \|^{2}+1)^{-1})
\end{aligned}
$$

\subsection{Uplink achievable rate}

\subsubsection{Uplink achievable rate with perfect CSI}

With perfect CSI, the uplink achievable rate of sensor $g_{u, k}^{e}$ can be written as

$$
\ddot{R}_{g_{u, k}^{e}}(\alpha)=(1-\alpha) \log _{2}\left(1+\ddot{\gamma}_{g_{u, k}^{e}}\right)
$$

where $\ddot{\gamma}_{g_{u, k}^{e}}$ denotes the SINR of the sensor $g_{u, k}^{e}$. Since perfect CSI is available, $\Xi_{g_{u, k}^{e}}$ in (32) can be rewritten as

$$
\begin{aligned}
& \Xi_{g_{u, k}^{e}}=\ddot{\Xi}_{g_{u, k}^{e}}+\sigma^{2} \mathbf{I}_{b_{u}^{e}} \\
& \left.=\sum_{g^{\prime}{ }_{u} \in G_{u} /\left\{g_{u}^{e}\right\}} \tilde{\mathbf{H}}_{g_{u}^{\prime}}^{\left\{B_{g_{u}^{e}}^{e}\right\}} \Lambda_{g^{\prime}{ }_{u}}^{1 / 2}\left(\tilde{\mathbf{H}}_{g_{u}^{\prime}}^{\left\{B_{u} e_{u}^{e}\right.}\right\} \Lambda_{g^{\prime}{ }_{u}}^{1 / 2}\right)^{H} \\
& +\sum_{g_{d}^{\prime} \in G_{d}} \tilde{\mathbf{H}}_{S I}^{\left\{B_{g_{u}^{e}}, B_{g_{d}^{\prime}}\right\}} \mathbf{W}_{g^{\prime} d} \Lambda_{g^{\prime} d}^{1 / 2}\left(\tilde{\mathbf{H}}_{S I}^{\left\{B_{g_{u}^{e}}^{e}, B_{g^{\prime}}\right\}} \mathbf{W}_{g^{\prime} d} \Lambda_{g^{\prime} d}^{1 / 2}\right)^{H} \\
& +\sigma^{2} \mathbf{I}_{b_{u}^{e}}
\end{aligned}
$$

and (32) is in generalized Rayleigh quotient form. The maximum value of (32) is obtained when

$$
\ddot{\mathbf{W}}_{g_{u, k}^{e}}=\frac{\left(\ddot{\Xi}_{g_{u, k}^{e}}+\sigma^{2} \mathbf{I}_{b_{u}^{e}}\right)^{-1} \tilde{\mathbf{h}}_{g_{u, k}^{e}}\left\{B_{g_{u}^{e}}\right\}}{\left\|\left(\ddot{\Xi}_{g_{u, k}^{e}}+\sigma^{2} \mathbf{I}_{b_{u}^{e}}\right)^{-1} \tilde{\mathbf{h}}_{g_{u, k}^{e}}^{\left\{B_{g_{u}^{e}}^{e}\right\}}\right\|}
$$

The maximum SINR of sensor $g_{u, k}^{e}$ can be expressed as

$$
\begin{aligned}
\ddot{\gamma}_{g_{u, k}^{e}} & =p_{g_{u, k}^{e}}\left(\tilde{\mathbf{h}}_{g_{u, k}^{e}}\left\{g_{g_{u}^{e}}\right\}\right)^{H}\left(\ddot{\Xi}_{g_{u, k}^{e}}+\sigma^{2} \mathbf{I}_{b_{u}^{e}}\right)^{-1} \tilde{\mathbf{h}}_{g_{u, k}^{e}}^{\left.B_{g_{u}^{e}}\right\}} \\
& =\frac{\alpha}{1-\alpha} \hat{p}_{g_{d, k}^{e}}^{H}\left(\tilde{\mathbf{h}}_{g_{u, k}^{e}}\left\{B_{g_{u}^{e}}\right\}\right)^{H}\left(\ddot{\Xi}_{g_{u, k}^{e}}+\sigma^{2} \mathbf{I}_{b_{u}^{e}}\right)^{-1} \tilde{\mathbf{h}}_{g_{u, k}^{e}}\left\{B_{g_{u}^{e}}\right\}
\end{aligned}
$$

With the help of Sherman-Morrison formula [59], the achievable rate of sensor $g_{u, k}^{e}$ can be rewritten as

$$
\left.\ddot{R}_{g_{u, k}^{e}}(\alpha)=(1-\alpha) \log _{2}\left(1+\frac{\alpha \hat{p}_{g_{d, k}^{e}}^{H}}{(1-\alpha) \sigma^{2}}\left(\| \tilde{\mathbf{h}}_{g_{u, k}^{e}}^{B_{g_{u}^{e}}^{e}}\right\} \|^{2}-\vartheta_{g_{u, k}^{e}}\right)\right)
$$

where $\vartheta_{g_{u, k}^{e}}$ can be expressed as

$$
\vartheta_{g_{u, k}^{e}}=\frac{\left(\tilde{\mathbf{h}}_{g_{u, k}^{e}}^{\left\{B_{g_{u}^{e}}\right\}}\right)^{H} \ddot{\Xi}_{g_{u, k}^{e}} \tilde{\mathbf{h}}_{g_{u, k}^{e}}^{\left\{B_{g_{u}^{e}}\right\}}}{\sigma^{2}+\operatorname{tr}\left(\ddot{\Xi}_{g_{u, k}^{e}}\right)}
$$

In this way, the achievable rate of sensor $g_{u, k}^{e}$ can be further rewritten as

$$
\ddot{R}_{g_{u, k}^{e}}(\alpha)=(1-\alpha) \log _{2}\left(1+\frac{\alpha \hat{p}_{g_{d, k}^{e}}^{H}}{(1-\alpha) \sigma^{2}}\left\|\tilde{\mathbf{h}}_{g_{u, k}^{e}}^{\left\{B_{g_{u}}\right\}}\right\|^{2}\right)
$$

when $\ddot{\Xi}_{g_{u, k}^{e}}$ is completely eliminated, and hence, we have $\vartheta_{g_{u, k}^{e}}=0$.

\subsubsection{Uplink achievable rate with imperfect CSI}

According to (29) and using the bounding technique in [58], the average achievable rates at the downlink user $g_{u, k}$ can be expressed as (44) at the top of the next page. The transmit power of sensor $g_{d, k}^{e}$ is set to $p_{g_{u, k}^{e}}=p_{g_{d, k}^{e}}^{\mathrm{H}}$. The powers of CEE, IUI, IGI, and SI are explained in the equation. 


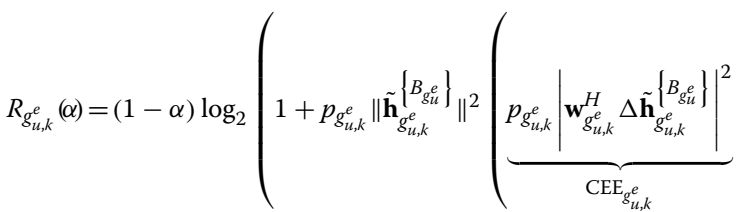

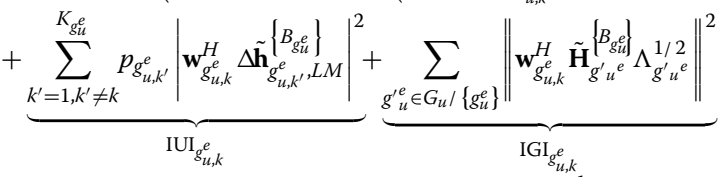

$$
\begin{aligned}
& +\underbrace{\sum_{g_{d}^{\prime} \in G_{d}}\left\|\mathbf{w}_{g_{u, k}^{e}}^{H} \tilde{\mathbf{H}}_{S I}^{\left\{B_{g_{u}^{e}}^{e}, B_{g_{d}^{\prime}}\right\}} \mathbf{w}_{g_{d}^{\prime}} \Lambda_{g_{d}^{\prime}}^{1 / 2}\right\|^{2}}_{\operatorname{SI}_{g_{u, k}^{e}}}+\left\|\mathbf{w}_{g_{u, k}^{e}}^{e}\right\|^{2})^{-1})
\end{aligned}
$$

\subsection{Optimization of the achievable sum rate}

In particular system, the assumption of having perfect instantaneous CSI is idealistic due to the fact that the CSI at the BS, users, and sensors are obtained by estimation or feedback. Hence, the CSI is subject to estimation, feedback, delay, and quantization errors. In this subsection, we consider the problem of optimizing the system achievable sum rate with imperfect CSI.

The achievable sum rate of the system with imperfect CSI can be written as

$$
R_{\mathrm{SUM}}(\alpha)=\sum_{g_{d, k} \in \mathbf{K}_{D}} R_{g_{d, k}}(\alpha)+\sum_{g_{u, k}^{e} \in \mathbf{K}_{U}} R_{g_{u, k}^{e}}(\alpha)
$$

where $\mathbf{K}_{D}$ denotes the set of all the users and $\mathbf{K}_{U}$ denotes the set of all the sensors. The downlink rate $R_{g_{d, k}}$ of user $g_{d, k}$ and the uplink rate $R_{g_{u, k}^{e}}$ of sensor $g_{u, k}^{e}$ are given in (36) and (44), respectively. The system achievable sum rate maximization problem can be written as

$$
\begin{aligned}
& \max _{\mathcal{P}, \alpha} \sum_{g_{d, k} \in \mathbf{K}_{D}} R_{g_{d, k}}(\alpha)+\sum_{g_{u, k}^{e} \in \mathbf{K}_{U}} R_{g_{u, k}^{e}}(\alpha) \\
& \text { s.t. }\left\{\begin{array}{l}
p 1: 1>\alpha \geq \tau_{u} / T \\
p 2: \sum_{d=1}^{K_{g}^{E}} p_{g_{d}^{e}} \leq P_{d}^{E} \\
p 3: \sum_{k=1}^{K_{d}} p_{g_{d, k}}\left\|\mathbf{w}_{g_{d, k}}\right\|^{2} \leq P_{d} \\
p 4: p_{g_{d, k}}^{e}, p_{g_{d, k^{\prime}}} \geq 0, k \in \mathbf{K}_{U}, k^{\prime} \in \mathbf{K}_{D}
\end{array}\right.
\end{aligned}
$$

where $K_{g}^{E}$ denotes the number of sensor groups. $P_{d}^{\mathrm{E}}$ and $P_{d}$ denote the maximum transmit power constraint at the BS in phase $I$ and phase $I I$, respectively. $\mathcal{P}=$ $\left\{p_{g_{d, 1}}, \cdots, p_{g_{d, K_{d}}}, p_{g_{1}^{e}}, \cdots, p_{g_{K_{g}^{E}}^{e}}\right\}$.

\subsubsection{Optimize $\mathcal{P}$ for fixed $\alpha$}

We assume that the length of the pilot signals and energy signals are fixed, that is, $\tau_{u}$ and $\tau_{d}$ are fixed. The downlink rate $R_{g_{d, k}}$ can be written as $R_{g_{d, k}}=(1-$ $\alpha) \log _{2}\left(1+\gamma_{g_{d, k}}\right)$, and the SINR at user $g_{d, k}$ can be written as $\gamma_{g_{d, k}}=\frac{p_{g_{d, k}} \mathbb{E}\left[\left[\Upsilon_{g_{d}}^{-1}\right]_{k, k}\right]}{\mathrm{CEE}_{g_{d, k}}+\mathrm{IUI}_{g_{d, k}}+\mathrm{IGI} g_{d, k}+1}$. The uplink rate $R_{g_{u, k}^{e}}$ can be written as $R_{g_{u, k}^{e}}=(1-\alpha) \log _{2}\left(1+\gamma_{g_{u, k}^{e}}\right)$, and the SINR of user $g_{u, k}^{e}$ signal can be written as $\gamma_{g_{u, k}^{e}}=$ $p_{g_{u, k}^{e}} b_{g_{u, k}^{e}}\left(c_{g_{u, k}^{e}}+d_{g_{u, k}^{e}}\right)^{-1}$, wherein $p_{g_{u, k}^{e}}=\frac{\alpha}{(1-\alpha)} p_{g_{d}^{e}} q_{g_{d, k}^{e}}$ and $b_{g_{u, k}^{e}}^{e}, c_{g_{u, k}^{e}}^{e}, d_{g_{u, k}^{e}}$ are positive variables and are unrelated to $\alpha$. Specifically, $b_{g_{u, k}^{e}}=\left\|\tilde{\mathbf{h}}_{g_{u, k}^{e}}^{\left\{B_{g_{u}}\right\}}\right\|^{2}, c_{g_{u, k}^{e}}=\mathrm{CEE}_{g_{u, k}^{e}}+$ $\mathrm{IUI}_{g_{u, k}^{e}}+\mathrm{IGI}_{g_{u, k}^{e}}$, and $d_{g_{u, k}^{e}}=\mathrm{SI}_{g_{u, k}^{e}}+\left\|\mathbf{w}_{g_{u, k}^{e}}^{H}\right\|^{2}$. We omit the user group index, and the sum rate optimization problem can be rewritten as

$$
\begin{aligned}
& \max _{\mathcal{P}} \prod_{k=1}^{K_{u}}\left(1+\gamma_{u, k}^{e}\right) \prod_{k=1}^{K_{d}}\left(1+\gamma_{d, k}\right) \\
& =\min _{\mathcal{P}}\left[\prod_{k=1}^{K_{u}}\left(1+\gamma_{u, k}^{e}\right) \prod_{k=1}^{K_{d}}\left(1+\gamma_{d, k}\right)\right]^{-1} \\
& \text { s.t. }\left\{\begin{array}{l}
c 1: \gamma_{g_{u, k}^{e}} \leq \frac{\alpha}{(1-\alpha)} q_{g_{d, k}^{e}} p_{g_{d}^{e}} b_{g_{u, k}^{e}}\left(c_{g_{u, k}^{e}}+d_{g_{u, k}^{e}}\right)^{-1}, k=1, \cdots, K_{u} \\
c 2: \gamma_{g_{d, k}} \leq \frac{p_{g_{d, k}} \mathbb{E}\left[\left[\Upsilon_{g_{d}}^{-1}\right]_{k, k}\right]}{\mathrm{CEE}_{g_{d, k}}+\mathrm{IUI}_{g_{d, k}}+\mathrm{IGI} I_{d, k}+1}, k=1, \cdots, K_{u} \\
p 1, p 2, p 3, p 4
\end{array}\right.
\end{aligned}
$$

where $p 1, p 2, p 3$, and $p 4$ are power constraints given by (46). The form of SE optimization problem in (47) is also close to a geometric programming (GP) except that the target function is not in the posynomial form [60]. In this paper, we apply the technique in [61] to approximate the target and solve it with the help of convex optimization tools. Specifically, $1+\gamma_{a, k}$ can be approximated by $\lambda_{a, k} \gamma_{a, k}^{\mu_{a, k}}$ close to a point $\tilde{\gamma}_{a, k}$, where $\mu_{a, k}=$ $\tilde{\gamma}_{a, k}\left(1+\tilde{\gamma}_{a, k}\right)^{-1}, \lambda_{a, k}=\tilde{\gamma}_{a, k}^{-\mu_{a, k}}\left(1+\tilde{\gamma}_{a, k}\right)$, and $a \in\{u, d\}$. As a result, (47) can be rewritten as

$$
\begin{aligned}
& \min _{P} \prod_{k=1}^{K_{u}}\left(\lambda_{u, k}^{e}\right)^{-1}\left(\gamma_{u, k}^{e}\right)^{-\mu_{u, k}} \prod_{k=1}^{K_{d}}\left(\lambda_{d, k}\right)^{-1} \gamma_{d, k}^{-\mu_{d, k}} \\
& \text { s.t. }\left\{\begin{array}{l}
c 1: \gamma_{g_{u, k}^{e}}\left(c_{g_{u, k}^{e}}+d_{g_{u, k}^{e}}\right)\left(\frac{\alpha}{(1-\alpha)} q_{g_{d, k}^{e}} p_{g_{d}^{e}} b_{g_{u, k}^{e}}\right)^{-1} \leq 1, k=1, \cdots, K_{u} \\
c 2: \frac{\gamma_{g_{d, k}}\left(\mathrm{CEE}_{g_{d, k}}+\mathrm{IUI}_{g_{d, k}}+\mathrm{IGI}_{g_{d, k}}+1\right)}{p_{g_{d, k}}\left[\left[\Upsilon_{g_{d}}^{-1}\right]_{k, k}\right]} \leq 1, k=1, \cdots, K_{d} \\
p 1, p 2, p 3, p 4
\end{array}\right.
\end{aligned}
$$


Constraints $c 1$ and $c 2$ can be rewritten as (49) and (50) at the top of this page.

$$
\begin{aligned}
& c 1: \frac{1}{\left\|\tilde{\mathbf{h}}_{g_{u, k}^{e}}^{\left.B_{g_{u}}\right\}}\right\| \|^{2}}\left(\gamma g_{u, k}^{e}\left|\mathbf{w}_{g_{u, k}^{e}}^{H} \Delta \tilde{\mathbf{h}}_{g_{u, k}^{e}, L M}\left\{B_{g_{u}^{e}}^{e}\right\}\right|^{2}\right. \\
& +\gamma_{g_{u, k}^{e}} p_{g_{u, k}^{e}}^{-1} \sum_{k^{\prime}=1, k^{\prime} \neq k}^{K_{g_{u}}^{e}} p_{g_{u, k^{\prime}}^{e}} \mid \mathbf{w}_{g_{u, k}^{e}}^{H} \Delta \tilde{\mathbf{h}}_{g_{u, k^{\prime}}^{e}}^{\{}\left\{\left.\begin{array}{c}
\left.B_{g_{u}^{e}}^{e}\right\} \\
\left.\right|^{\prime}
\end{array}\right|^{2}\right. \\
& +\gamma_{g_{u, k} e} p_{g_{u, k}^{e}}^{-1} \sum_{g_{u}^{\prime e} \in G_{u} /\left\{g_{u}^{e}\right\}} \sum_{k^{\prime}=1}^{K_{g_{u}^{\prime}}^{e}} p_{g_{u, k^{\prime}}^{\prime e}}\left|\mathbf{w}_{g_{u, k}^{e}}^{H} \tilde{\mathbf{h}}_{g_{u, k^{\prime}}^{\prime}}^{\left.B_{g_{u}^{e}}\right\}}\right|^{2} \\
& +\gamma_{g_{u, k}^{e}}^{e} p_{g_{u, k}^{e}}^{-1} \sum_{g_{d}^{\prime} \in G_{d}} \sum_{k^{\prime}=1}^{K_{g_{d}^{\prime}}} p_{g_{d, k^{\prime}}^{\prime}}\left|\mathbf{w}_{g_{u, k}^{e}}^{H} \tilde{\mathbf{H}}_{S I}^{\left\{B_{g_{u}^{e}}^{e}, B_{g_{d}^{\prime}}\right\}} \mathbf{w}_{g_{d, k^{\prime}}^{\prime}}\right|^{2} \\
& \left.+\gamma_{g_{u, k}^{e}} p_{g_{u, k}^{e}}^{-1}\left\|\mathbf{w}_{g_{u, k}^{e}}^{H}\right\|^{2}\right) \leq 1 \\
& k=1, \cdots, K_{u} \\
& c 2: \frac{1}{\mathbb{E}\left[\left[\Upsilon_{g_{d}}^{-1}\right]_{k, k}\right]}\left(\gamma_{g_{d, k}}\left|\left(\Delta \tilde{\mathbf{h}}_{g_{d, k}, L M}^{\left\{\beta_{g_{d}}\right\}}\right)^{H} \mathbf{w}_{g_{d, k}}\right|^{2}\right. \\
& +\gamma_{g_{d, k}} \sum_{g_{d, k}}^{-1} \sum_{k^{\prime}=1, k^{\prime} \neq k}^{K_{g_{d}}} p_{g_{d, k^{\prime}}}\left|\left(\Delta \tilde{\mathbf{h}}_{g_{d, k}, L M}\left\{B_{g_{d}}\right\}\right)^{H} \mathbf{w}_{g_{d, k^{\prime}}}\right|^{2} \\
& \left.+\gamma_{g_{d, k}} p_{g_{d, k}}^{-1} \sum_{g_{d}^{\prime} \in G_{d l} \mid\left\{g_{d}\right\}} \sum_{k^{\prime}=1}^{K_{g_{d}^{\prime}}} p_{g_{d, k^{\prime}}^{\prime}}\left|\left(\tilde{\mathbf{h}}_{g_{d, k}}\left\{B_{g_{d}^{\prime}}\right\}\right)^{H} \mathbf{w}_{g_{d, k^{\prime}}^{\prime}}^{H}\right|^{2}+\gamma_{g_{d, k}} p_{g_{d, k}}^{-1}\right) \leq 1 \\
& k=1, \cdots, K_{d}
\end{aligned}
$$

The SE optimization problem in (48) becomes a standard GP and can be solved by the Algorithm 1.

\subsubsection{Optimize $\alpha$ for fixed $\mathcal{P}$}

Since the downlink rate $R_{g_{d, k}}(\alpha)$ can be rewritten as $R_{g_{d, k}}(\alpha)=(1-\alpha) \zeta_{g_{d, k}}$, where $\zeta_{g_{d, k}}=$ $\log _{2}\left(1+\frac{p_{d, k} \mathbb{E}\left[\left[\Upsilon_{g_{d}}^{-1}\right]_{k, k}\right]}{\mathrm{CEE}_{g_{d, k}}+\mathrm{IUI}_{g_{d, k}}+1}\right)$ is a variable unrelated to $\alpha$. The ratio used for information transmission can be adjusted by changing the during of phase II, that is $(1-\alpha) T$. The uplink rate $R_{g_{u, k}^{e}}(\alpha)$ can be rewritten as

$$
R_{g_{u, k}^{e}}(\alpha)=(1-\alpha) \log _{2}\left(1+\gamma_{g_{u, k}^{e}}(\alpha)\right)
$$

where $\gamma_{g_{u, k}^{e}}(\alpha)=\frac{\alpha \tilde{b}_{g_{u, k}^{e}}}{(1-\alpha)}\left(\frac{\alpha \tilde{g}_{u, k}^{e}}{(1-\alpha)}+d_{g_{u, k}^{e}}\right)^{-1}$ and $\tilde{b}_{g_{u, k}^{e}}, \tilde{c}_{g_{u, k}^{e}}, d_{g_{u, k}^{e}}$ are positive variables and are unrelated to $\alpha$. Specifically, $\tilde{b}_{g_{u, k}^{e}}=q_{g_{d, k}^{e}} p_{g_{d}^{e}}\left\|\tilde{\mathbf{h}}_{g_{u, k}^{e}}^{\left\{B_{g_{u}^{e}}\right\}}\right\|^{2}$, $\tilde{c}_{g_{u, k}^{e}}=\left(\mathrm{CEE}_{g_{u, k}^{e}}+\mathrm{IUI}_{g_{u, k}^{e}}+\mathrm{IGI}_{g_{u, k}^{e}}\right)(1-\alpha) / \alpha$, and

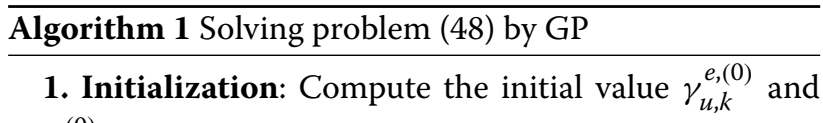
$\gamma_{d, k}^{(0)}, i=1$, set the value of tolerance $\varepsilon$ and maximum iteration number $L$.

2. For the $n$th iteration:

- Compute $\mu_{u, k}=\gamma_{u, k}^{(n)}\left(1+\gamma_{u, k}^{(n)}\right)^{-1}$,

$\mu_{d, k}=\gamma_{d, k}^{(n)}\left(1+\gamma_{d, k}^{(n)}\right)^{-1}$,

$\lambda_{u, k}^{e}=\left(\gamma_{u, k}^{e,(n)}\right)^{-\mu_{u, k}}\left(1+\gamma_{u, k}^{e,(n)}\right)$ and

$\lambda_{d, k}=\left(\gamma_{d, k}^{(n)}\right)^{-\mu_{d, k}}\left(1+\gamma_{d, k}^{(n)}\right)$.

- Solve problem (48) by GP:

$$
\begin{aligned}
& \min _{P} \prod_{k=1}^{K_{u}}\left(\lambda_{u, k}^{e}\right)^{-1}\left(\gamma_{u, k}^{e}\right)^{-\mu_{u, k}} \prod_{k=1}^{K_{d}}\left(\lambda_{d, k}\right)^{-1} \gamma_{d, k}^{-\mu_{d, k}} \\
& \text { s.t. } c 1, c 2, p 1, p 2, p 3, p 4
\end{aligned}
$$

3. If $\max _{k=1, \cdots, K_{u}}\left|\gamma_{u, k}^{e,(i)}-\gamma_{u, k}^{e,(i-1)}\right|<\varepsilon$ and $\max _{k=1, \cdots, K_{d}}\left|\gamma_{d, k}^{(i)}-\gamma_{d, k}^{(i-1)}\right|<\varepsilon$, stop. Else, set $n=n+1$ and go back to step 2 .

$d_{g_{u, k}^{e}}=\mathrm{SI}_{g_{u, k}^{e}}+1$ are variables unrelated to $\alpha$. The achievable sum rate maximization problem of $\alpha$ can be rewritten as

$$
\begin{aligned}
\max _{\alpha} R_{\mathrm{SUM}}(\alpha)= & (1-\alpha) \sum_{g_{d, k} \in \mathbf{K}_{D}} \zeta_{g_{d, k}} \\
& +\sum_{g_{u, k}^{e} \in \mathbf{K}_{U}}(1-\alpha) \log _{2}\left(1+\gamma_{g_{u, k}^{e}}(\alpha)\right)
\end{aligned}
$$

s.t.1 $>\alpha \geq \tau_{u} / T$

Let $\zeta=\sum_{g_{d, k} \in \mathbf{K}_{D}} \zeta_{g_{d, k}}$ and (52) can be rewritten as

$$
\begin{aligned}
\max _{\alpha} R_{\mathrm{SUM}}(\alpha)= & (1-\alpha) \zeta \\
& +\sum_{g_{u, k}^{e} \in \mathbf{K}_{U}}(1-\alpha) \log _{2}\left(1+\gamma_{g_{u, k}^{e}}(\alpha)\right) \\
\text { s.t.1 }>\alpha \geq \tau_{u} / T &
\end{aligned}
$$

The partial derivative of function $R_{\mathrm{SUM}}(\alpha)$ can be expressed as

$$
\begin{aligned}
\frac{\partial R_{\mathrm{SUM}}(\alpha)}{\partial \alpha}= & -\zeta-\sum_{g_{u, k}^{e} \in \mathbf{K}_{U}} \log _{2}\left(1+\gamma_{g_{u, k}^{e}}(\alpha)\right) \\
& +(1-\alpha) \sum_{g_{u, k}^{e} \in \mathbf{K}_{u} \ln (2)\left(1+\gamma_{g_{u, k}^{e}}(\alpha)\right)\left(\alpha \tilde{c}_{g_{u, k}^{e}}^{e}+(1-\alpha) d_{g_{u, k}^{e}}\right)^{2}}
\end{aligned}
$$


The two order derivative of function $R_{\mathrm{SUM}}(\alpha)$ can be expressed as (55) at the top of this page.

$$
\begin{aligned}
& \frac{\partial^{2} R_{\mathrm{SUM}}(\alpha)}{\partial \alpha^{2}}=\sum_{g_{u, k}^{e} \in \mathbf{K}_{U}} \frac{2 \tilde{b}_{g_{u, k}} d_{g_{u, k}^{e}}}{\ln (2)\left(1+\gamma_{g_{u, k}}(\alpha)\right)\left(\alpha \tilde{c}_{g_{u, k}^{e}}+(1-\alpha) d_{g_{u, k}}\right)^{2}}
\end{aligned}
$$

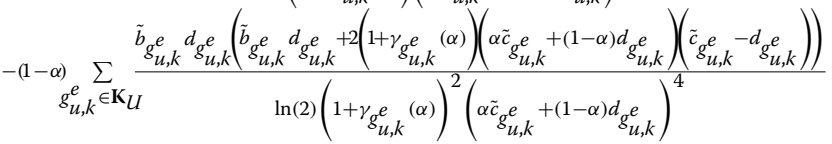

Consistent with the assumption in [52], in this paper, we assume that the power of CEE, IUI, and IGI is much larger than SI and AWGN. Hence, we have $\frac{\partial^{2} R_{\mathrm{SUM}}(\alpha)}{\partial^{2} \alpha}<0$ for $0<\alpha<1$ and we can obtain the optimum $\alpha$ of $R_{\mathrm{SUM}}(\alpha)$ when $\frac{\partial R_{\mathrm{SUM}}(\alpha)}{\partial \alpha}=0$, that is

$$
\begin{aligned}
& \underbrace{(1-\alpha)^{2} \sum_{g_{u, k}^{e} \in \mathbf{K}_{U}} \frac{\tilde{b}_{g_{u, k}^{e}} \ln (2)\left(1+\gamma_{g_{u, k}^{e}}\right.}{\left.g_{u, k}(\alpha)\right)\left(\alpha \tilde{c}_{g_{u, k}^{e}}+(1-\alpha) d_{g_{u, k}^{e}}\right)^{2}}}_{A(\alpha)} \\
& =(1-\alpha) \zeta+(1-\alpha) \sum_{g_{u, k}^{e} \in \mathbf{K}_{U}} \log _{2}\left(1+\gamma_{g_{u, k}^{e}}(\alpha)\right) \\
& =\left.R_{\mathrm{SUM}}(\alpha)\right|_{\mathcal{P}}
\end{aligned}
$$

Equation (56) is a transcendental equation, and we also can solve it numerically or resort the Newton iterative method to solve the problem, that is, $\hat{\alpha}_{k+1}=\hat{\alpha}_{k}-$ $\frac{\partial R_{\mathrm{SUM}}(\alpha)}{\partial \alpha} /\left.\frac{\partial^{2} R_{\mathrm{SUM}}(\alpha)}{\partial^{2} \alpha}\right|_{\alpha=\hat{\alpha}_{k}}$. Let $\hat{\alpha}^{\mathrm{Opt}}$ denote the solution of (56) and if $1>\alpha \mathrm{Opt}>\tau_{u} / T$, then the solution of the problem in (53) is $\alpha^{\mathrm{Opt}}$. Moreover, it is interesting to see that the maximum sum rate of the system is $R_{\mathrm{SUM}}^{\mathrm{OPT}}=A\left(\alpha^{\mathrm{Opt}}\right)$. Otherwise, the solution of the problem in (53) is $\tau_{u} / T$, and the maximum sum rate of the system is

$$
R_{\mathrm{SUM}}^{\mathrm{Opt}}=\sum_{g_{d, k} \in \mathbf{K}_{D}} R_{g_{d, k}}\left(\tau_{u} / T\right)+\sum_{g_{u, k}^{e} \in \mathbf{K}_{U}} R_{g_{u, k}^{e}}\left(\tau_{u} / T\right)
$$

\subsubsection{Joint optimization of $\alpha$ and $\mathcal{P}$}

The joint optimal $\alpha$ and $\mathcal{P}$ can be obtained by finding the optimum $\mathcal{P}$ for each $\alpha$ and then selecting the found $\mathcal{P}$ and $\alpha$ that maximize the problem in (46). Hence, a onedimensional search over $\alpha$ is needed. We can conclude that the required one-dimensional search can be limited to a small region of $\alpha$ by exploiting the structure of the problem (46) and the properties obtained in (54)-(56). Hence, the computational complexity for solving the joint optimization problem can be greatly reduced. The proposed solution to jointly optimize $\alpha$ and $\mathcal{P}$ is summarized in Algorithm 2.

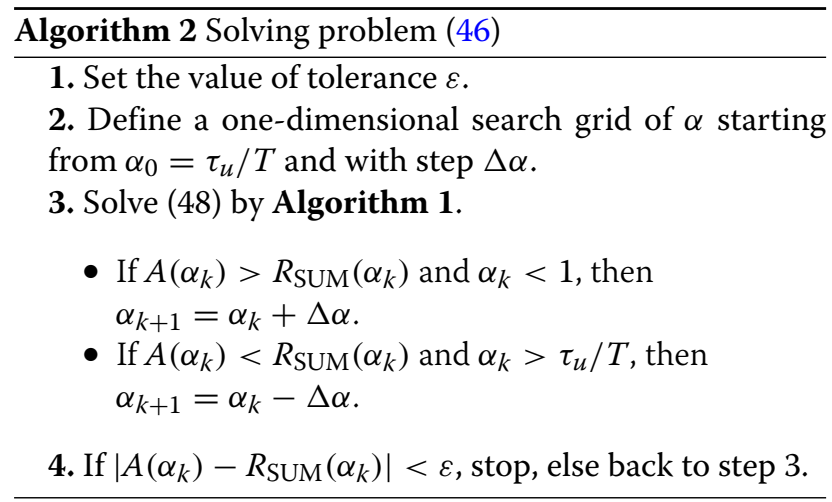

\section{Simulation}

In this section, the performance of BD hybrid TS and PS SWIPT protocol in FD massive MIMO system is evaluated using the 3GPP LTE simulation model for macro-cell environment [62]. The center frequency is $2.4 \mathrm{GHz}$ and system bandwidth is set to $20 \mathrm{MHz}$. Thermal noise density is set to $-174 \mathrm{dBm} / \mathrm{Hz}$. The channel coherent time is 200 symbol times. The path loss between BS and users/sensors are modeled as $2.7+42.8 \log _{10}(R)(\mathrm{dB})$, and the path loss between users/sensors are modeled as $55.78+40 \log _{10}(R)$ (dB), where $R$ denotes the distance. The number of scattering clusters is set to $M_{u}=M_{d}=1$. It is assumed that the passive SIC scheme for infrastructure nodes proposed in [63] has been employed at the BS. In such scheme, the suppression is from two parts, namely (1) the path loss introduced by the $20 \mathrm{~m}$ separation between transmit and receive antenna arrays and (2) an additional cancelation of $45 \mathrm{~dB}$ provided by techniques, such as radio frequency absorber material and cross-polarization. No other active SIC scheme is used in this paper.

In this paper, we assume that the BS can obtain the perfect DOA and DOD information when users access the system. In the following simulations, we consider the fixed sensors, hence it is reasonable to assume that the BS can obtain the perfect DOA and DOD information of sensors. We first consider a scenario where the sensors and users gather perfectly in three groups and the DOA/DOD regions of users in each group are identical. We assume that each group contains 5 uplink/downlink users. The DOA/DOD regions of three user groups are $\left[-45^{\circ},-35^{\circ}\right],\left[12^{\circ}, 22^{\circ}\right]$ and $\left[42^{\circ}, 52^{\circ}\right]$, respectively. Since we assume $M_{u}=1$, the DOA region of uplink group is $[a, b]$, that is $\left[\theta_{g_{u, k}, 1}^{\min }, \theta_{g_{u, k}, 1}^{\max }\right]=[a, b]$. Similarly, the DOA/DOD regions of three sensor groups are $\left[-42^{\circ},-32^{\circ}\right],\left[16^{\circ}, 26^{\circ}\right]$ and $\left[39^{\circ}, 49^{\circ}\right]$, respectively. The DOA and DOD regions of SI channel between the transmit antenna array and receive antenna array are set to $\left[-12^{\circ},-22^{\circ}\right],\left[56^{\circ}, 66^{\circ}\right]$ and $\left[-56^{\circ},-46^{\circ}\right],\left[27^{\circ}, 37^{\circ}\right]$, respectively. The resulting ABSs for all the groups satisfy the user and sensor grouping criteria. 
Figure 4 illustrates the SE of the proposed BD hybrid TS and PS SWIPT protocol for FD massive MIMO system with perfect user and sensor grouping. The transmit powers of BS is $20.2 \mathrm{dBm}$. The distance between users/sensors and $\mathrm{BS}$ is set to $500 \mathrm{~m}$. This setup ensures the average downlink receive SNR is $3 \mathrm{~dB}$. The energy conversion efficiency $\eta$ is set to 0.8 , and the power ratio used for energy harvesting $\beta$ is set to 0.8 . For comparison, existing SWIPT protocols designed for massive MIMO (MM) system are also demonstrated, such as TDD SWIPT with MF precoder in [49], TDD SWIPT with ZF precoder in [50], and TDD SWIPT with MRT precoder in [51]. Meanwhile, traditional FDD MM with JSDM [7], FD MM with linear transceiver [64], FD MM with spatial SI suppression [17], and traditional BD FD MM transmission scheme [52] are also extended to the SWIPT scenario and demonstrated for comparison. Note that the FD MM with spatial SI suppression in [17], the BS requires instantaneous CSI of SI channel in order to perform SI cancelation in spatial domain. In the simulations, the uplink transmit power in $[7,17,52,64]$ are also harvested from the BS downlink transmit signal. With estimated effective BD CSI, the SE of the proposed BD hybrid TS and PS SWIPT protocol outperforms the existing transmission schemes. The reason is that, the proposed BD hybrid TS and PS SWIPT transmission scheme can reduce the required length of pilot sequence significantly, hence significant SE gain can be achieved by the proposed scheme over the TDD and FDD MM systems and traditional FD MM. The SE of the proposed BD hybrid TS and PS SWIPT protocol with perfect CSI and the capacity are also depicted in Fig. 4.

In Fig. 5, we test the robustness of the proposed BD hybrid TS and PS SWIPT protocol for FD MM system under the presence of DOA/DOD offset. We can see from Fig. 5 that the proposed BD hybrid TS and PS SWIPT protocol obtains the best performance. Moreover, the SE performance decreases with the increasing of DOA/DOD offset. This is because the proposed BD SWIPT protocol requires accurate DOA/DOD information when users and sensors are partitioned into groups. For the traditional SWIPT protocols in [49-51], linear precoders are adopted so that the user/sensor DOA/DOD information is not required.

In Fig. 6, the SE performance of the proposed BD hybrid TS and PS SWIPT protocol for FD MM system with different PS ratio $\beta$ is demonstrated. In Fig. 7, the SE performance of the proposed BD hybrid TS and PS SWIPT protocol for FD MM system with different energy conversion efficiency $\eta$. We can see from Figs. 6 and 7 that the proposed BD hybrid TS and PS SWIPT protocol obtains the best performance.

Figure 8 depicts the SE of the proposed BD hybrid TS and PS SWIPT protocol for FD MM system with imperfect user and sensor grouping. In Fig. 8, there are 50 users and 50 sensors within the coverage of BS which is equipped with $N=128$ transmit/receive antennas. The signal of each user/sensor is within a $10^{\circ} \mathrm{DOA} / \mathrm{DOD}$ region which is randomly distributed in the sectors. The

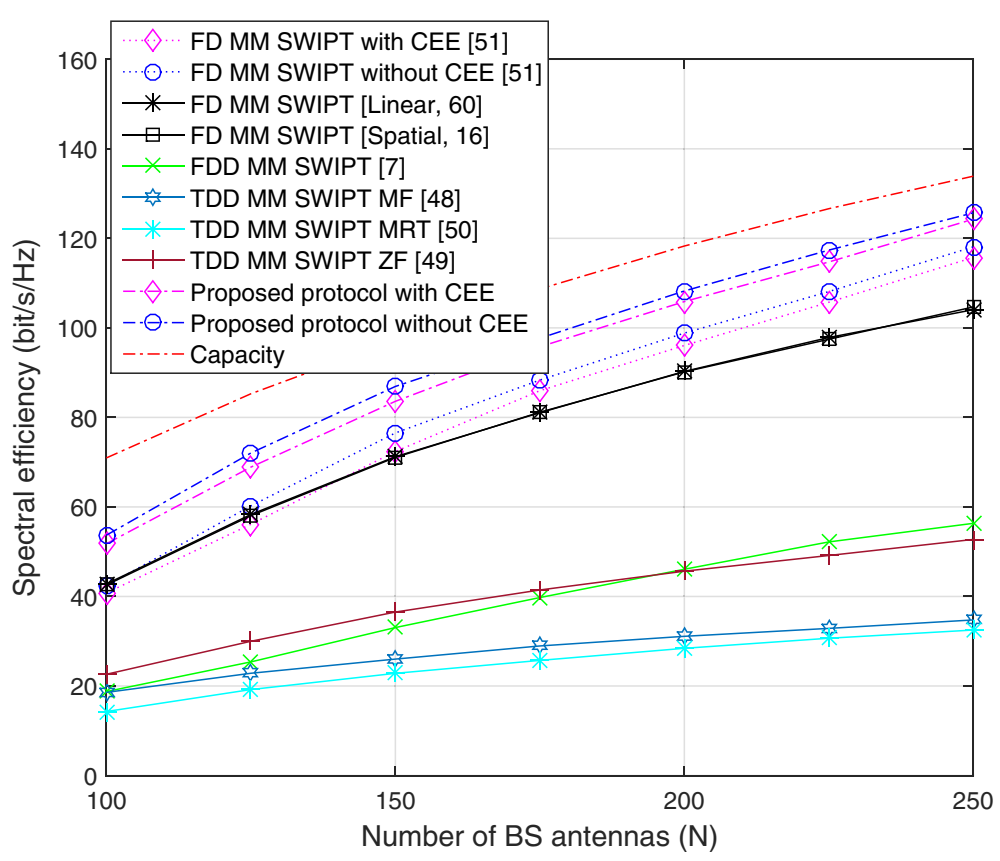

Fig. 4 SE of the proposed BD hybrid TS and PS SWIPT protocol for FD MM system with perfect user and sensor grouping, $\beta=0.8, \eta=0.8$ 


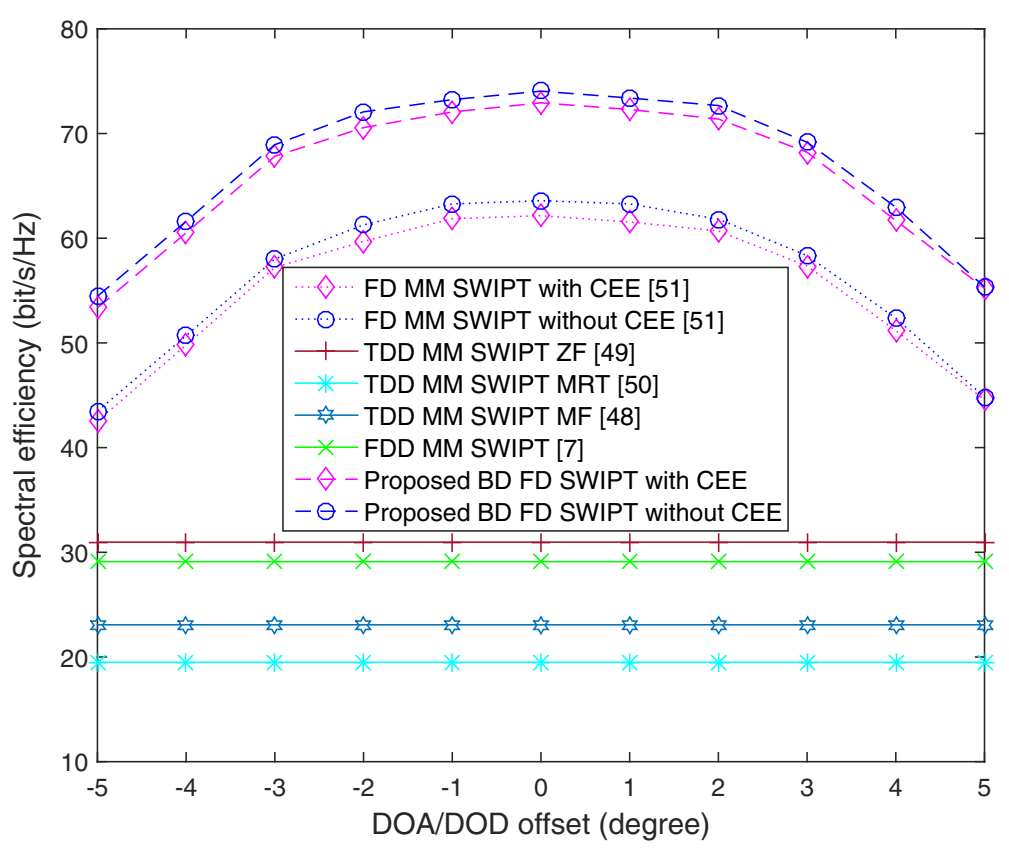

Fig. 5 SE of the proposed BD hybrid TS and PS SWIPT protocol for FD massive MIMO system with different DOA/DOD offset, $N=128, \beta=0.8, \eta=0.8$

distance between uplink/downlink UE and BS is randomly distributed in the interval $[200,1000]$ meters. After user and sensor grouping, three user groups and three sensor groups are formed, and the users and sensors in different groups are allocated orthogonal time-frequency resources. Since users and sensors are grouped into three groups, respectively, the SE is defined as the average SEs for three groups. The number of scattering cluster for SI channel is set to $M_{S I}=2$. We can see from Fig. 8 that the proposed BD hybrid TS and PS SWIPT protocol for FD MM system obtains the best performance. For the FD MM with linear transceiver [64], we assume that there is no

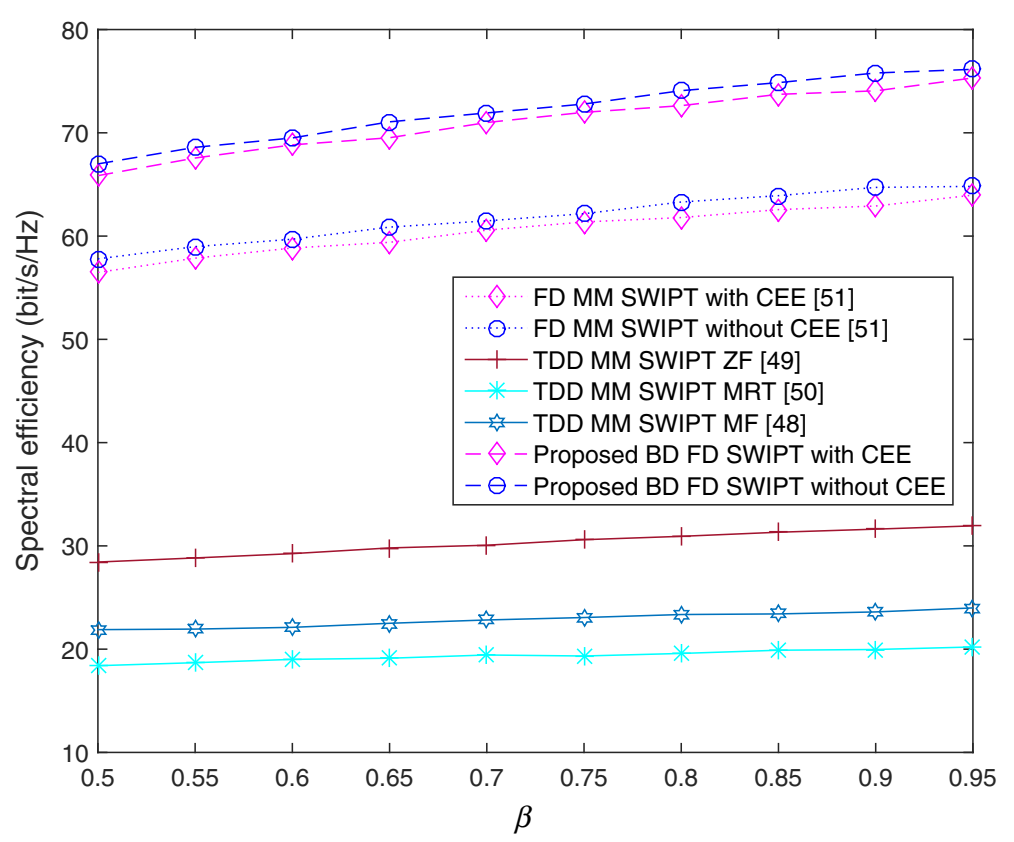

Fig. 6 SE of the proposed BD hybrid TS and PS SWIPT protocol for FD massive MIMO system with different PS ratio $\beta, N=128, \eta=0.8$ 


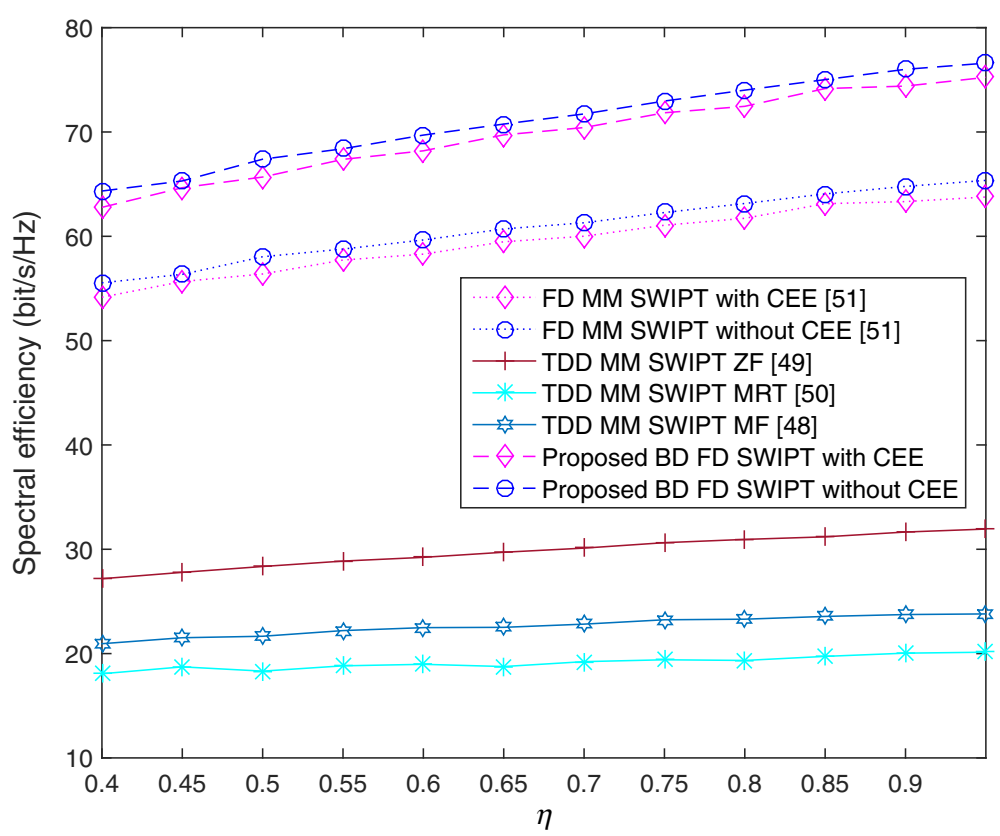

Fig. 7 Spectral efficiency of the proposed beam-domain hybrid TS and PS SWIPT protocol for FD massive MIMO system with different energy conversion efficiency $\eta, N=128, \beta=0.8$

downlink reciprocity, hence the SE performance is greatly reduced as a result of the heavy cost of pilot resources.

Figure 9 depicts the SE of the proposed BD hybrid TS and PS SWIPT protocol for FD MM system under the condition that the number of scattering clusters MSI is increase from 2 to 10 . The SI signal from each scattering cluster is within a $10^{\circ} \mathrm{DOA} / \mathrm{DOD}$ region which is randomly distributed $\left[-90^{\circ}, 90^{\circ}\right]$. Interestingly, with the increase of the number of scattering clusters $M_{\mathrm{SI}}$, the SE of the proposed BD hybrid TS and PS SWIPT

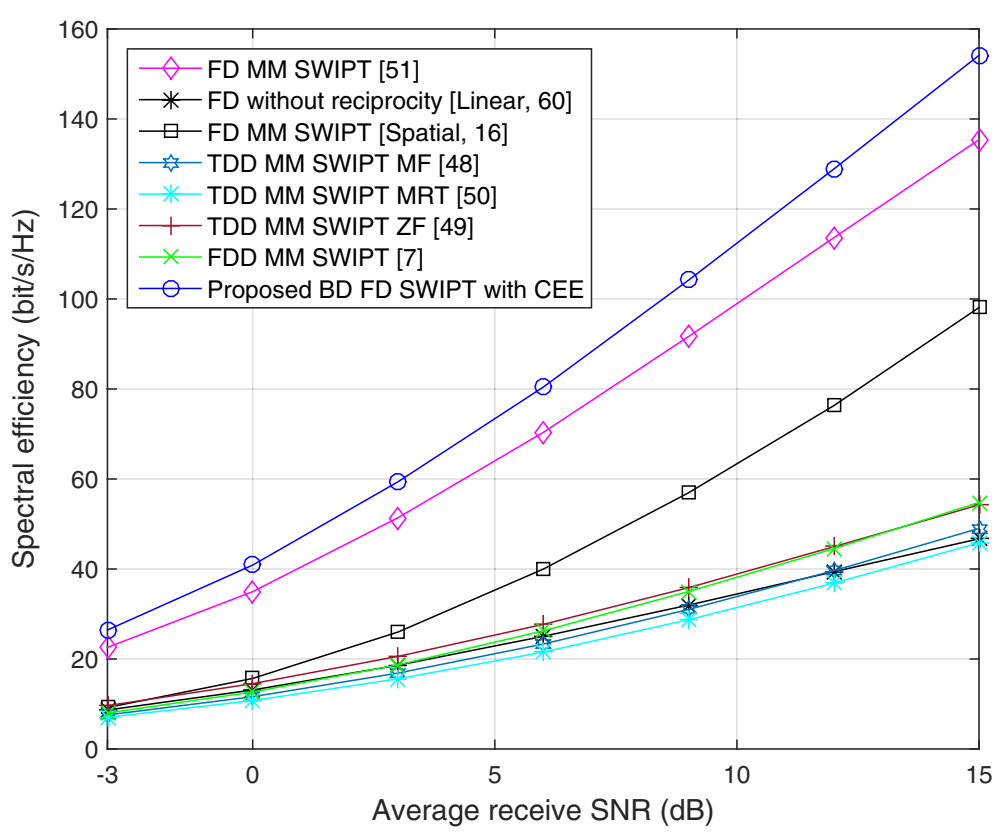

Fig. 8 SE of the proposed BD hybrid TS and PS SWIPT protocol for FD massive MIMO system with imperfect user and sensor grouping, $N=128, \beta=0.8, \eta=0.8$ 


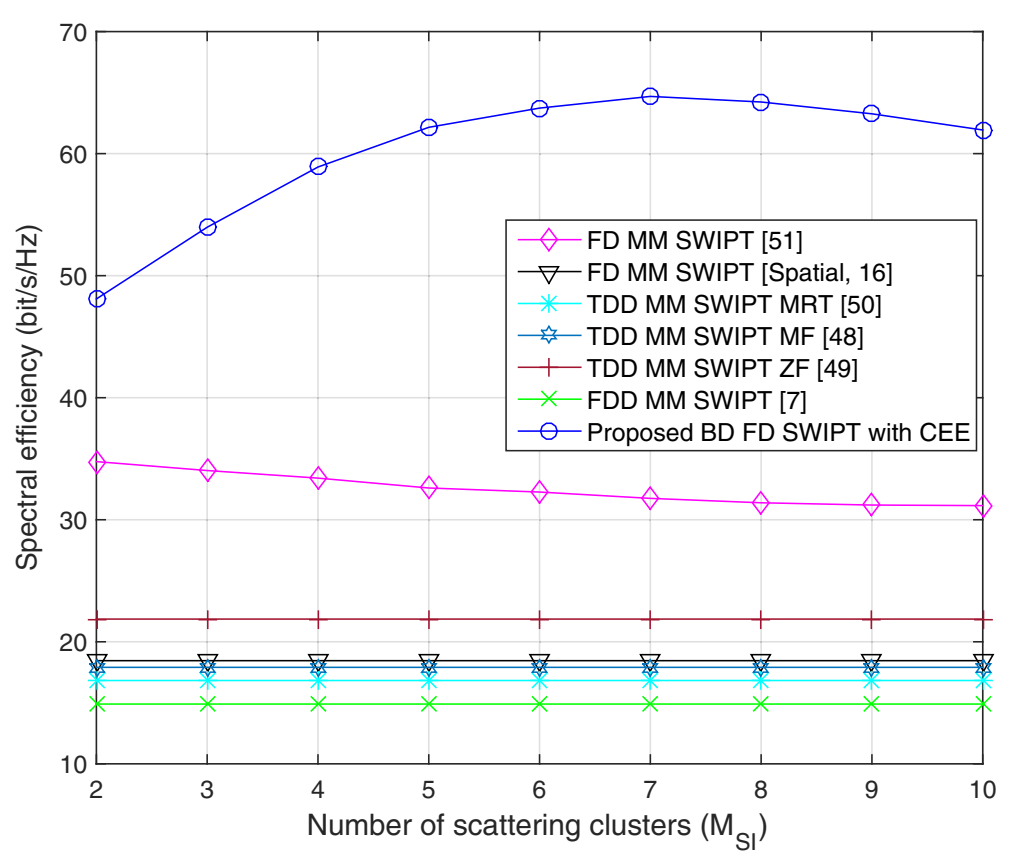

Fig. 9 SE of the proposed BD hybrid TS and PS SWIPT protocol for FD massive MIMO system with different numbers of scattering clusters MSI Over SI channel, $N=128, \beta=0.8, \eta=0.8$

protocol for FD MM transmission scheme increases first and then decreases when $M_{\mathrm{SI}}>6$. Meanwhile, the proposed BD hybrid TS and PS SWIPT protocol for FD MM transmission scheme obtains the best SE performance. The SE of FD MM scheme in [52] approaches to that of the TDD/FDD MM as $M_{S I}$ increases. This is because the numbers of uplink groups and the active beams for each uplink group decrease as $M_{\mathrm{SI}}$ increases.

\section{Conclusions}

In this paper, we propose a BD hybrid TS and PS SWIPT protocol for FD massive MIMO system. In order to reduce the pilot resource cost used for channel estimation, we resort BEM to represent massive MIMO channel in the BD. Due to the spatial sparsity of massive MIMO channel, we can compress the massive MIMO channel in the BD under certain selected basis spaces and the channel dimension required to be estimated can be greatly reduced. The hybrid TS and PS SWIPT protocol which lies in intelligently scheduling the users and sensors based on the distributions of their associated BD ABSs to mitigate SI and enhance transmission efficiency is designed. The whole BD hybrid TS and PS SWIPT protocol is divided into two phases based on the ideal of TS. The first phase is used for cellular users uplink training and sensor nodes energy harvesting as well as downlink training, wherein the cellular users transmit uplink pilots for uplink channel estimation at the BS, while the BS simultaneously transmits energy signals to the sensor nodes. Based on the idea of PS, the sensor nodes utilize the received energy signals for energy harvesting and downlink channel estimation. In the second phase, the BS forms the transmit beamformers for information transmission to the users as well as the receive beamformers for the sensors transmit their data to the BS simultaneously. By optimizing the TS ratio and transmit powers at the BS in two phases, the system achievable sum rate performance is maximized. Simulation results shown the superiority of the proposed protocol on SE compared with the existing massive MIMO SWIPT protocol.

\section{Acknowledgements}

The authors would like to thank the reviewers for their careful readings and valuable comments.

\section{Funding}

This work is supported by the Jiangsu Province Natural Science Foundation under grant BK20160079, National Natural Science Foundation of China (no. 61671472).

\section{Authors' contributions}

KX proposed the main idea and is the main writer of this paper. ZS, YW, and XX assisted in the simulations and analysis. All authors read and approved the final manuscript.

Competing interests

The authors declare that they have no competing interests.

\section{Publisher's Note}

Springer Nature remains neutral with regard to jurisdictional claims in published maps and institutional affiliations. 
Received: 16 June 2017 Accepted: 30 January 2018 Published online: 12 February 2018

\section{References}

1. TL Marzetta, Noncooperative cellular wireless with unlimited numbers of BS antennas. IEEE Trans. Wirel. Commun. 9(11), 3590-3600 (2010)

2. F Rusek, D Persson, BK Lau, EG Larsson, TL Marzetta, O Edfors, F Tufvesson, Scaling up MIMO: opportunities and challenges with very large arrays. IEEE Signal Process. Mag. 30(1), 40-60 (2013)

3. J Hoydis, S Brink, M Debbah, Massive MIMO in UL/DL of cellular networks: how many antennas do we need? IEEE J. Sel. Areas Commun. 31, 160-171 (2013)

4. EG Larsson, F Tufvesson, O Edfors, TL Marzetta, Massive MIMO for next generation wireless systems. IEEE Commun. Mag. 52(2), 186-195 (2014)

5. F Boccardi, RW Heath Jr, A Lozano, TL Marzetta, P Popovski, Five disruptive technology directions for 5G. IEEE Commun. Mag. 52(2), 74-80 (2014)

6. PWC Chan, ES Lo, RR Wang, EKS Au, VKN Lau, RS Cheng, WH Mow, RD Murch, KB Letaief, The evolution path of $4 \mathrm{G}$ networks: FDD or TDD? IEEE Commun. Mag. 44(12), 42-50 (2006)

7. A Adhikary, J Nam, J-Y Ahn, G Caire, Joint spatial division and multiplexing: the large-scale array regime. IEEE Trans. Info. Theory. 59(10), 6441-6463 (2013)

8. C Sun, X Gao, S Jin, M Matthaiou, Z Ding, C Xiao, Beam division multiple access transmission for massive MIMO communications. IEEE Trans. Commun. 63(6), 2170-2184 (2015)

9. A Liu, V Lau, Phase only RF precoding for massive MIMO systems with limited RF chains. IEEE Trans. Signal Process. 62(17), 4505-4515 (2014)

10. D Kim, G Lee, Y Sung, Two-stage beamformer design for massive MIMO downlink by trace quotient formulation. IEEE Trans. Commun. 63(6), 2200-2211 (2015)

11. A Sabharwal, P Schniter, D Guo, DW Bliss, S Rangarajan, R Wichman, In-band full-duplex wireless: challenges and opportunities. IEEE J.Sel. Areas Commun. 32(9), 1637-1652 (2014)

12. D Kim, H Lee, D Hong, A survey of in-band full-duplex transmission: from the perspective of PHY and MAC layers. IEEE Commun. Surv. Tutor. 17(4), 2017-2046 (2015). Fourthquarter

13. Z Zhang, X Chai, K Long, AV Vasilakos, L Hanzo, Full duplex techniques for $5 G$ networks: self-interference cancellation, protocol design, and relay selection. IEEE Commun. Mag. 53(5), 128-137 (2015)

14. Y Liao, K Bian, L Song, Z Han, Full-duplex MAC protocol design and analysis. IEEE Commun. Lett. 19(7), 1185-1188 (2015)

15. U Ugurlu, T Riihonen, R Wichman, Optimized in-band full-duplex MIMO relay under single-stream transmission. IEEE Trans. Veh. Technol. 65(1), 155-168 (2016)

16. Y Jang, K Min, S Park, S Choi, in 2015 IEEE International Conference on Communications (ICC). Spatial resource utilization to maximize uplink spectral efficiency in full-duplex massive MIMO, (London, 2015), pp. $1583-1588$

17. HA Suraweera, I Krikidis, G Zheng, C Yuen, PJ Smith, Low-complexity end-to-end performance optimization in MIMO full-duplex relay systems. IEEE Trans. Wirel. Commun. 13(2), 913-927 (2014)

18. Z Liu, W Du, D Sun, Energy and spectral efficiency tradeoff for massive MIMO systems with transmit antenna selection. IEEE Trans. Veh. Technol. 66(5), 4453-4457 (2017)

19. Y Li, P Fan, A Leukhin, Liu L, On the spectral and energy efficiency of full-duplex small-cell wireless systems with massive MIMO. IEEE Trans. Veh. Technol. 66(3), 2339-2353 (2017)

20. S Sudevalayam, P Kulkarni, Energy harvesting sensor nodes: survey and implications. IEEE Commun. Surv. Tutor. 13(3), 443-46 (2011)

21. L Chen, FR Yu, H Ji, B Rong, X Li, VCM Leung, Green full-duplex self-backhaul and energy harvesting small cell networks with massive MIMO. IEEE J. Sel. Areas Commun. 34(12), 3709-3724 (2016)

22. DK Nguyen, DNK Jayakody, S Chatzinotas, JS Thompson, J Li, in IEEE Access, vol. 5. Wireless energy harvesting assisted two-way cognitive relay networks: protocol design and performance analysis, (2017), pp. 21447-21460

23. W Wang, R Wang, H Mehrpouyan, N Zhao, G Zhang, in IEEE Access, vol. 5. Beamforming for simultaneous wireless information and power transfer in two-way relay channels, (2017), pp. 9235-9250

24. MA Marsan, G Bucalo, AD Caro, M Meo, Y Zhang, in 2013 IEEE International Conf. on Communications Workshops (ICC). Towards zero grid electricity networking: powering BSs with renewable energy sources, (Budapest, 2013), pp. 596-601

25. H Liu, KJ Kim, KS Kwak, HV Poor, QoS-constrained relay control for full-duplex relaying with SWIPT. IEEE Trans. Wireless Commun. 16(5), 2936-2949 (2017)

26. G Pan, H Lei, Y Yuan, Z Ding, Performance analysis and optimization for SWIPT wireless sensor networks. IEEE Trans. Commun. 65(5), 2291-2302 (2017)

27. F Zhou, Z Li, J Cheng, Q Li, J Si, Robust AN-aided beamforming and power splitting design for secure MISO cognitive radio with SWIPT. IEEE Trans. Wirel. Commun. 16(4), 2450-2464 (2017)

28. AA Lu, X Gao, YR Zheng, C Xiao, in IEEETransactions on Communications, vol. 65, no. 7. Linear precoder design for SWIPT in MIMO broadcasting systems with discrete input signals: manifold optimization approach, (2017), pp. 2877-2888

29. YH Suh, K Chang, A high-efficiency dual-frequency rectenna for 2.45-and 5.8-GHz wireless power transmission. IEEE Trans. Microw. Theory Tech. 50(7), 1784-1789 (2002)

30. JR Smith, Wirelessly powered sensor networks and computational RFID. (Springer-Verlag New York, New York, 2013)

31. K Huang, V Lau, Enabling wireless power transfer in cellular networks: architecture, modeling and deployment. IEEE Trans. Wirel. Commun. 13(2), 902-912 (2014)

32. LR Varshney, in Proc. IEEE International Symposium on Information Theory (ISIT). 2008 Transporting information and energy simultaneously, (Toronto, 2008), pp. 1612-1616

33. P Grover, A Sahai, in 2010 IEEE International Symposium on Information Theory. Shannon meets Tesla: wireless information and power transfer, (Austin, 2010), pp. 2363-2367

34. X Zhou, R Zhang, CK Ho, Wireless information and power transfer: architecture design and rate-energy tradeoff. IEEE Trans. Commun. 61(11), 4754-4767 (2013)

35. X Lu, P Wang, D Niyato, DI Kim, Z Han, Wireless networks with RF energy harvesting: a contemporary survey. IEEE Commun. Surv. Tutor. 17(2), 757-789 (2015)

36. K Huang, $X$ Zhou, Cutting the last wires for mobile communications by microwave power transfer. IEEE Commun. Mag. 53(6), 86-93 (2015)

37. MM Zhao, Y Cai, Q Shi, B Champagne, MJ Zhao, Robust transceiver design for MISO interference channel with energy harvesting. IEEE Trans. Signal Process. 64(17), 4618-4633 (2016)

38. I Krikidis, S Timotheou, S Sasaki, RF energy transfer for cooperative networks: data relaying or energy harvesting? IEEE Commun. Lett. 16(11), 1772-1775 (2012)

39. AA Nasir, X Zhou, S Durrani, RA Kennedy, Relaying protocols for wireless energy harvesting and information processing. IEEE Trans. Wirel. Commun. 12(7), 3622-3636 (2013)

40. Y Cai, MM Zhao, Q Shi, B Champagne, MJ Zhao, Joint transceiver design algorithms for multiuser MISO relay systems with energy harvesting. IEEE Trans. Commun. 64(10), 4147-4164 (2016)

41. Z Chen, B Xia, H Liu, in 2014 IEEE Global Conference on Signal and Information Processing (GlobalSIP). Wireless information and power transfer in two-way amplify-and-forward relaying channels, (Atlanta, 2014), pp. 168-172

42. KTutuncuoglu, B Varan, A Yener, Throughput maximization for two-way relay channels with energy harvesting nodes: the impact of relaying strategies. IEEE Trans. Commun. 63(6), 2081-2093 (2015)

43. D Li, C Shen, Z Qiu, in 2013 IEEE International Conference on Communications (ICC). Two-way relay beamforming for sum-rate maximization and energy harvesting, (Budapest, 2013), pp. 3155-3120

44. AA Okandeji, MRA Khandaker, KK Wong, Z Zheng, in 2016 IEEE Globecom Workshops (GC Wkshps). Joint transmit power and relay two-way beamforming optimization for energy-harvesting full-duplex communications, (Washington, 2016), pp. 1-6

45. L Zhang, Y Cai, M Zhao, B Champagne, L Hanzo. Nonlinear MIMO transceivers improve wireless-powered and self-interference-aided relaying, vol. 16, (2017), pp. 6953-6966

46. MM Zhao, Q Shi, Y Cai, MJ Zhao, Joint transceiver design for full-duplex cloud radio access networks with SWIPT. IEEE Trans. Wirel. Commun 16(9), 5644-5658 (2017)

47. MM Zhao, Y Cai, Q Shi, M Hong, B Champagne, Joint transceiver designs for full-duplex $K$-pair MIMO interference channel with SWIPT. IEEE Trans. Commun. 65(2), 890-905 (2017) 
48. W Wu, B Wang, Y Zeng, H Zhang, Z Yang, Z Deng, Robust secure beamforming for wireless powered full-duplex systems with self-energy recycling. IEEE Trans.Veh. Technol. 66(11) (10055-10069)

49. L Fan, H Zhang, Y Huang, L Yang, Exploiting BS sntenna tilt for SWIPT in 3-D massive MIMO systems. IEEE Wirel. Commun. Lett. 6(5), 666-669 (2017)

50. X Wang, J Liu, C Zhai, in IEEE Transactions on Wireless Communications, vol. 16, no. 11. Wireless power transfer based multi-pair two-way relaying with massive antennas, (2017), pp. 7672-7684

51. S Lee, Y Zeng, R Zhang, in IEEE Wireless Communications Letters, vol. PP, no. 99. Retrodirective multi-user wireless power transfer with massive MIMO, (2017), pp. 1-1

52. X Xia, KXu, D Zhang, Y Xu, Y Wang, Beam-domain full-duplex massive MIMO: realizing co-time co-frequency uplink and downlink transmission in the cellular system. IEEE Trans. Veh. Technol. 66(10), 8845-8862 (2017)

53. 3GPP TR 25.996, Universal mobile telecommunications system (UMTS): spatial channel model for multiple input multiple output (MIMO) simulations, v.12.0.0 (2012). www.3gpp.org. Accessed 07 Feb 2018

54. KI Pedersen, PE Mogensen, BH Fleury, A stochastic model of the temporal and azimuthal dispersion seen at the base station in outdoor propagation environments. IEEE Trans.Veh. Technol. 49(2), 437-447 (2000)

55. J Singh, S Ramakrishna, On the feasibility of codebook-based beamforming in millimeter wave systems with multiple antenna arrays. IEEE Trans.Wirel. Commun. 14(5), 2670-2683 (2015)

56. GB Giannakis, C Tepedelenlioglu, Basis expansion models and diversity techniques for blind identification and equalization of timevarying channels. Proc. IEEE. 86(10), 1969-1986 (1998)

57. D Nguyen, LN Tran, et al., Precoding for full duplex multiuser MIMO systems: spectral and energy efficiency maximization. IEEE Trans. Signal Process. 61(16), 4038-4050 (2013)

58. B Hassibi, BM Hochwald, How much training is needed in multiple-antenna wireless links? IEEE Trans. Inf. Theory. 49(4), 951-963 (2003)

59. Hager WW, Updating the inverse of a matrix. SIAM Rev. 31, 221-239 (1989)

60. S Boyd, L Vandenberghe, Convex Optimization. (Cambridge University Press, University Printing House, Cambridge, 2004)

61. PC Weeraddana, M Codreanu, M Latva-aho, A Ephremides, Resource allocation for cross-layer utility maximization in wireless networks. IEEE Trans. Veh. Technol. 60(6), 2790-2809 (2011)

62. 3GPP TR 36.828, Further enhancements to LTE time division duplex (TDD) for downlink-uplink (DL-UL) interference management and traffic adaptation, v.11.0.0 (2012). www.3gpp.org. Accessed 07 Feb 2018

63. E Everett, A Sahai, A Sabharwal, Passive self-interference suppression for full-duplex infrastructure nodes. IEEE Trans. Wirel. Commun. 24(2), 680-694 (2014)

64. HQ Ngo, HA Suraweera, M Matthaiou, EG Larsson, Multipair full-duplex relaying with massive arrays and linear processing. IEEE J. Sel. Areas Commun. 32(9), 1721-1737 (2014)

\section{Submit your manuscript to a SpringerOpen ${ }^{\odot}$ journal and benefit from:}

- Convenient online submission

- Rigorous peer review

- Open access: articles freely available online

- High visibility within the field

- Retaining the copyright to your article

Submit your next manuscript at $\gg$ springeropen.com 